\title{
Photovoltaic Power Quality Analysis Based on the Modulation Broadband Mode Decomposition Algorithm
}

\author{
Zucheng Wang ${ }^{1}{ }^{\oplus}$, Yanfeng Peng ${ }^{1, *} \mathbb{C}$, Yanfei Liu ${ }^{2}$, Yong Guo ${ }^{1}$, Yi Liu ${ }^{3}$, Hongyan Geng ${ }^{1}$, Sai Li ${ }^{1}$ and Chao Fan ${ }^{1}$ \\ 1 Hunan Provincial Key Laboratory of Health Maintenance for Mechanical Equipment, \\ Hunan University of Science and Technology, Xiangtan 411201, China; chengzuw@163.com (Z.W.); \\ hnkjdx_guoy@163.com (Y.G.); ghy7650453@163.com (H.G.); 21010301016@mail.hnust.edu.cn (S.L.); \\ 13250960210@163.com (C.F.) \\ 2 College of Mechanical and Vehicle Engineering, Hunan University, Changsha 410082, China; \\ lyfhndx@163.com \\ 3 National Innovation Center of Advanced Rail Transit Equipment, Zhuzhou 412000, China; \\ liuyi_hust@163.com \\ * Correspondence: pyf1988@sina.com or hndxpyf@hnu.edu.cn
}

Citation: Wang, Z.; Peng, Y.; Liu, Y.; Guo, Y.; Liu, Y.; Geng, H.; Li, S.; Fan, C. Photovoltaic Power Quality Analysis Based on the Modulation Broadband Mode Decomposition Algorithm. Energies 2021, 14, 7948. https://doi.org/10.3390/en14237948

Academic Editor: Teuvo Suntio

Received: 4 November 2021

Accepted: 24 November 2021

Published: 27 November 2021

Publisher's Note: MDPI stays neutral with regard to jurisdictional claims in published maps and institutional affiliations.

Copyright: (c) 2021 by the authors. Licensee MDPI, Basel, Switzerland. This article is an open access article distributed under the terms and conditions of the Creative Commons Attribution (CC BY) license (https:/ / creativecommons.org/licenses/by/ $4.0 /)$.

\begin{abstract}
The Broadband Mode Decomposition (BMD) method was previously proposed to solve the Gibbs phenomenon that occurs during photovoltaic signal decomposition; its main idea is to build a dictionary which contains signal features, and to search in the dictionary to solve the problem. However, BMD has some shortcomings; especially if the relative bandwidth of the decomposed signal is not small enough, it may treat a square wave signal as several narrowband signals, resulting in a deviation in the decomposition effect. In order to solve the problem of relative bandwidth, the original signal is multiplied by a high-frequency, single-frequency signal, and the wideband signal is processed as an approximate wideband signal. This is the modulation broadband mode decomposition algorithm (MBMD) proposed in this article. In order to further identify and classify the disturbances in the photovoltaic direct current (DC) signal, the experiment uses composite multiscale fuzzy entropy (CMFE) to calculate the components after MBMD decomposition, and then uses the calculated value in combination with the back propagation (BP) neural network algorithm. Simulation and experimental signals verify that the method can effectively extract the characteristics of the square wave component in the DC signal, and can successfully identify various disturbance signals in the photovoltaic DC signal.
\end{abstract}

Keywords: modulated broadband mode decomposition; BP neural network; signal feature extraction; disturbance identification; photovoltaic power quality

\section{Introduction}

The application of photovoltaic technology is becoming more and more widespread in daily life, and the generation industry of photovoltaic power is at a stage of rapid development. In photovoltaic systems, including DC and alternating current (AC) systems, there have been considerable economic benefits from the wide use of various related equipment such as frequency converters, inverters, rectifiers, and charging piles. However, in the power grid, many power quality problems such as harmonic, noise, and distortion issues will be produced due to the imbalance, impact, and nonlinearity of the load in the circuit. The use of electrical appliances and the stability of the power supply network will be seriously affected by these power quality problems [1-3]. Reference [4] proposes an evaluation scheme for renewable solar energy, which is aimed at the risk-benefit assessment of historical and traditional buildings. In terms of the photovoltaic direct current, the identification and classification of disturbance in photovoltaic DC signals is the key and basis for power quality analysis, evaluation, and management $[5,6]$, therefore the classification and identification of power quality disturbance has high research value. Reference [5] proposed 
a comprehensive solution, which is a real-time analysis of power quality disturbances, and reference [6] proposed a new type of framework for the recognition of complex power quality disturbances; this framework is based on the multiple fusion convolutional neural network (MFCNN), which could automatically extract and fuse signal features from multiple sources.

In the engineering field, in order to extract features from signals that contain noise, many time-frequency analysis methods [7-10] are used. Reference [7] proposed a new method of emitter identification, which is based on spectral features and variational mode decomposition. In order to monitor family activities, reference [8] proposed a feature extraction method of acoustic signals, which is based on the non-negative matrix factorization (NMF) classifier. Reference [9] is a very classical algorithm, namely the empirical mode decomposition method (EMD), which was proposed by N. E. Huang et al. This method and the subsequent improved algorithm played a key part in signal feature extraction and signal decomposition. In order to extract time-frequency information features from vibration signals, wavelet packet transform was applied in reference [10]. These adaptive time-frequency analysis methods for signal analysis can be roughly divided into two categories according to whether they are based on the Fourier transform. For example, the variational mode decomposition (VMD) algorithm is based on the Fourier transform. All of these methods realize signal decomposition by computing in the frequency domain. The essence of the VMD algorithm is an adaptive filter [11], and the method was proposed by D. Zosso and K. Dragomiretskiy. VMD shows good performance [12-14] compared with previous methods, especially in the analysis of complex, non-stationary signals, which has been proved in many references. Reference [12] proposed a novel method of online chatter identification for milling processes. In order to make a prediction of short-term wind speed, reference [13] combined linear and nonlinear prediction models and proposed a hybrid method based on variational mode decomposition. To detect the blade imbalance fault in the marine current turbine (MCT), reference [14] proposed a novel VMD denoising method. The original signal was decomposed into several intrinsic mode functions, which was obtained by calculating the envelope of the extreme point; this is the essence of a method not based on the Fourier transform [15], such as the ensemble empirical mode decomposition (EEMD), the local mode decomposition (LMD), and the empirical mode decomposition (EMD) methods. The EEMD algorithm was proposed by $\mathrm{Z}$. Wu et al., and compared with EMD, EEMD improves the anti-noise ability of the EMD method by averaging the decomposition results and mixing different levels of white noise into the original signal [16]. The VMD and EEMD methods are superior and effective [17,18], which can be shown by recent studies. Hence, the EEMD method was selected as a representative of the methods that are based on the non-Fourier transform, while the VMD method was selected as a representative of the methods that are based on the Fourier transform; these two algorithms were compared with the algorithm proposed in this paper.

Due to the incorporation and disconnection of linear loads in the circuit, an interference signal similar to a square wave signal is generated in the original photovoltaic DC signal, and this type of signal is generally sharp. Since signals with sharp corners, such as sawtooth signals and square wave signals, have frequency bands that are infinite and can be considered as wideband signals, the existing time-frequency analysis methods are expected show the Gibbs phenomenon when processing this kind of signal.

First, the essence of the algorithm based on the Fourier transform is multiscale adaptive filtering. However, after filtering, a series of interference may be caused at the breakpoint of the decomposition result; the characteristics of the sharp corner of the broadband signal disappearing or attenuating is known as the Gibbs phenomenon [19]. For broadband signals, the use of an improved Hilbert-Huang transform (HHT) and the energy entropy algorithm [20] to extract the features of alternating current (AC) signal square waves was proposed by K. He et al. Second, the essence of the methods that are based on the non-Fourier transform is the use of the interpolation function to calculate the envelope of the extreme point, and the division of the original signal into a number of intrinsic 
mode functions (IMFs) with a "smooth" narrow band. Therefore, when dealing with broadband components, errors will inevitably occur. Consequently, Y. Peng et al. proposed the Broadband Mode Decomposition (BMD) algorithm [21,22]. The innovative point of the BMD algorithm is to construct an association dictionary, which contains common broadband data such as narrowband data, square wave data, and sawtooth data. The sparse solution is then obtained by using the optimization method to search in the association dictionary. Compared with the previous signal decomposition algorithms, the BMD algorithm takes the adjustment differential operator as the optimal object; it can also extract signal features from complex signals, including narrowband signal features and broadband signal features. However, when applied to a wideband signal, the BMD algorithm may treat it as several narrowband components because its relative bandwidth of is not sufficiently small. Therefore, to denoise photovoltaic DC signals, a broadband mode decomposition (MBMD) method is proposed in this paper, which is based on the modulation differential operator. In order to make the decomposition result more accurate on the basis of the BMD algorithm, a high-frequency, single-frequency signal is multiplied by the MBMD so that the relative bandwidth of the effective wideband signal is converted into one that is sufficiently small and far lower than 1 , and the wideband signal is then regarded as an approximate wideband signal.

Recently, J. Zheng et al. proposed the composite multiscale fuzzy entropy (CMFE) algorithm, which is based on the multiscale fuzzy entropy (MFE) algorithm. It has been proved to be suitable for processing non-stationary signals in engineering [23,24]. Therefore, it is applied to construct the feature vectors of the MBMD decomposition results. Then, the $\mathrm{BP}$ neural network algorithm is used to identify and classify the photovoltaic DC current disturbance.

The remaining sections of the paper are as follows. Section 2 introduces the disturbance state in the photovoltaic DC current and establishes a photovoltaic DC mathematical model. Section 3 introduces the problems in the identification of photovoltaic DC disturbances. Section 4 describes the details of the MBMD algorithm. Section 5 explains the other algorithms used. Section 6 describes the simulation analysis of the feature extraction of the photovoltaic signal disturbance model. Section 7 analyzes the collected experimental data sets of photovoltaic direct current signals. Section 8 provides the conclusion. All the data in this paper come from the data collected by the photovoltaic experimental platform in the literature [25].

\section{Photovoltaic Electrical Signal Model}

According to the analysis of the measured signals, the mathematical model of the DC signal is initially established, which consists of the DC signal, a harmonic signal superimposed on the DC, a distortion signal caused by the impact load, a noise signal caused by external interference, electromagnetic interference, etc.

\subsection{Harmonic Signal}

In normal operation, the current of the DC system contains abundant harmonic signals. The harmonic signal refers to the AC component superimposed on the DC. Therefore, the established harmonic signal model is as follows:

$$
U_{1}(t)=k U_{0}(t) \sum_{i=1}^{n} \cos (2 \pi i \omega t) 0<k<0.1
$$

where, $U_{0}$ is the amplitude of the DC current, $k$ is the amplitude coefficient of harmonics, and $n$ is the maximum number of harmonics.

\subsection{Distortion Signal}

Due to the impact, nonlinearity, and imbalance of the load, various disturbance components in the photovoltaic DC system increase. These disturbance components are distortion signals. For the photovoltaic DC system, there are two kinds of disturbances 
that have the greatest impact on photovoltaic power quality: current mutation and current pulse. The sudden change of current refers to the steep rise and fall of the current caused when the load switch in the system is opened and closed. For linear loads such as light bulbs, when the load switch is opened or closed, the current change is mostly straight up and down; for nonlinear loads such as motors, when the load switch is closed or opened, the current changes are characterized by slopes that rise and fall. This is determined by the characteristics of the nonlinear load.

Therefore, the linear load current mutation signal model is established as follows:

$$
U_{2}(t)=U_{0}(t) \pm A\left[U_{0}\left(t-t_{1}\right)-U_{0}\left(t-t_{2}\right)\right]
$$

where $A$ is the amplitude coefficient of disturbance, $t_{1}$ is the initial time of disturbance, and $t_{2}$ is the end time of disturbance.

The nonlinear load current mutation signal model is the following:

$$
\begin{aligned}
U_{3}(t)=U_{0}(t)+ & {\left[k_{1} \times\left(t-t_{1}+b\right)^{2}+a_{1}\right]+\left[k_{2} \times\left(t-t_{2}+b\right)^{2}+a_{2}\right] } \\
+ & A\left[U_{0}\left(t-t_{1}-b\right)-U_{0}\left(t-t_{2}+b\right)\right]
\end{aligned}
$$

where $k_{1}$ and $k_{2}$ are the slope of the signal, $a_{1}$ and $a_{2}$ are the amplitudes of signal change, $b$ is the duration of the signal rise or fall, $t_{1}$ is the initial time of disturbance, and $t_{2}$ is the end time of disturbance.

A current pulse is produced when faults such as a grounding fault or an automatic restart occur in the operation of the photovoltaic DC system, and refers to the current generally rising quickly and then falling quickly with a large amplitude change, but with a relatively short duration, generally 10-20 ms.

Therefore, the current pulse signal model is established as:

$$
U_{4}(t)=U_{0}(t)+B \times\left[\sin 2 \pi \omega_{2}\left(t-t_{1}\right)-\sin 2 \pi \omega_{2}\left(t-t_{2}\right)\right]
$$

where $B$ is the amplitude coefficient of disturbance, $t_{1}$ is the initial time of disturbance, $t_{2}$ is the end time of disturbance, and $\omega_{2}$ is the frequency of the pulse signal.

\section{Problems in the Identification of Photovoltaic DC Disturbances}

\subsection{Gibbs Phenomenon}

The Fourier series expands a signal into the sum of sinusoidal signals. Taking square wave signals as an example, the Fourier expansion of broadband signals is as follows:

$$
\operatorname{square}(t)=\sum_{i=1}^{+\infty} \frac{1}{2 i-1} \sin [(2 i-1) t]
$$

Because the bandwidth of the filter is limited, the Gibbs phenomenon will appear after filtering. The Gibbs phenomenon is a phenomenon that may cause a series of disturbances at the breakpoint of the decomposition result, because the high frequency part of the broadband signal will attenuate or disappear after the filter is filtered. The wider the filter bandwidth, the more accurate the filter result is. Set square $(\omega t$, rate $)$ to be the square signal, where rate is the duty cycle and $\omega$ is the angular frequency. In Equation (6), the mixed signal $x_{1}(t)$ includes a noise signal with a signal-to-noise ratio (SNR) of 10 , a simple square signal, and an amplitude modulation and frequency modulation (AM-FM) sinusoidal signal. The time domain waveform of $x_{1}(t)$ and its components are shown in Figure 1.

$$
x_{1}(t)=\operatorname{square}(20 \pi t, 50)+0.5 \cos \left(10 \pi t+t^{2}\right) \sin (300 \pi t)+n(t)
$$




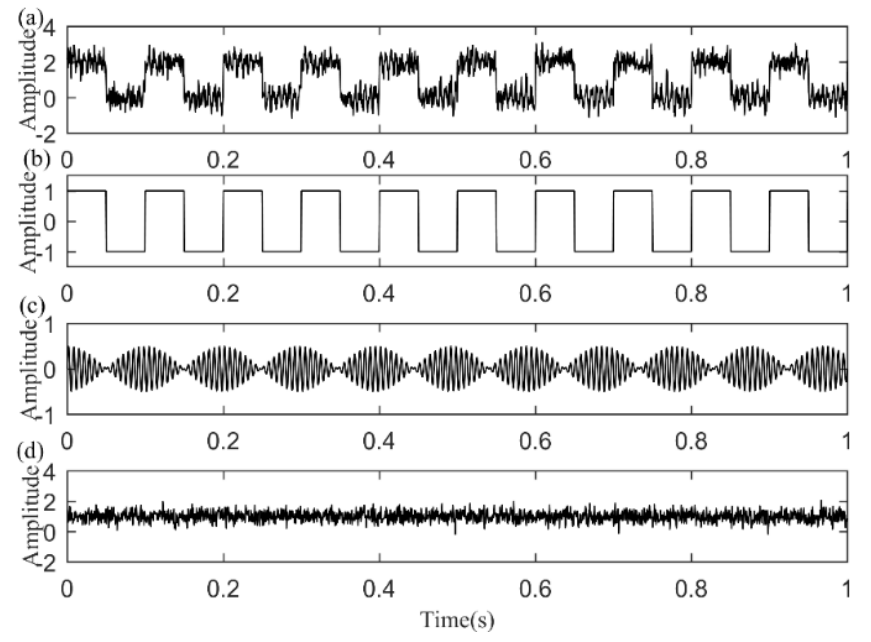

Figure 1. Time domain signals. (a) Mixed signals $x_{1}(t)$; (b) Square wave component; (c) AM-FM sine component; (d) Noise composition.

In order to obtain square wave components, the Butterworth bandpass filter is used to filter $x_{1}(t)$, and the result after filtering is shown in Figure 2. Through experiments, the optimized filter parameters are determined (passband cutoff frequency is $48 \mathrm{hz}$, stopband cutoff frequency is $90 \mathrm{hz}$ ). As can be seen in Figure 2a, after filtering, there is an inevitable disturbance in the result due to the Gibbs phenomenon.
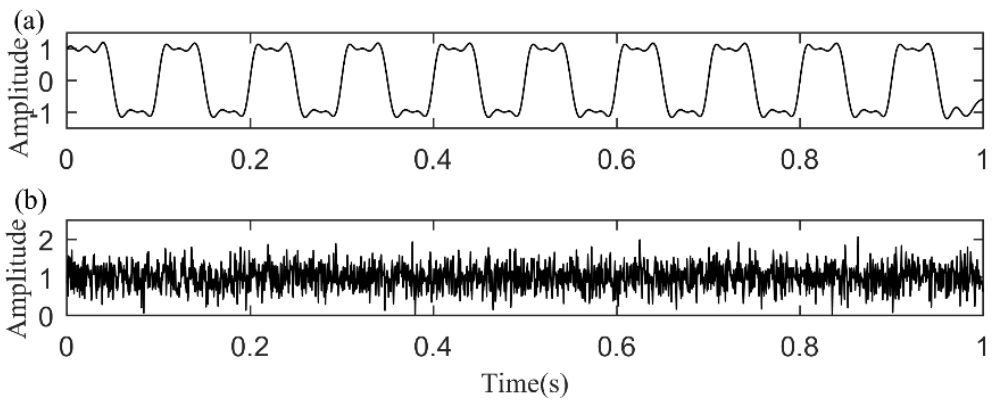

Figure 2. Filtering result of $x_{1}(t)$. (a) Square wave component; (b) Residues.

\subsection{VMD Algorithm}

By applying several Wiener filters, the VMD algorithm can adaptively divide the original signal into multiple components with limited frequency bands. By optimizing the variational model, the center frequency and bandwidth of the filter are obtained. The iterative process of the i-th IMF has the following three steps:

(1) Calculate the analytical formula of $I M F_{i}(t)$ through the Hilbert transform.

$$
\left[\delta(t)+\frac{j}{\pi t}\right] \times I M F_{i}(t)
$$

(2) Estimate the center frequency $\omega_{i}$ of the analyzed signal $I M F_{i}(t)$, and convert the spectrum of the analyzed signal into the frequency baseband corresponding to $\omega_{i}$.

$$
\left\{\left[\delta(t)+\frac{j}{\pi t}\right] \times I M F_{i}(t)\right\} e^{-j w i t}
$$

(3) Take the $L^{2}$ norm of the analytic signal gradient as the optimization objective function, and take its bandwidth as the optimization parameter. Obtain the decomposition 
results by the alternating direction multiplier method (ADMM) in the optimization process. Describe the optimization problem as follows:

$$
\min _{\left\{I M F_{i}(t)\right\},\left\{w_{i}\right\}} \sum_{i}\left\|\partial_{t}\left\{\left[\delta(t)+\frac{j}{\pi t}\right] \times I M F_{i}(t)\right\} e^{-j w_{i} t}\right\|^{2}, S . T . x(t)=\sum I M F_{i}(t)
$$

When analyzing broadband signals, the VMD algorithm will be affected by the Gibbs phenomenon because its essence is the multi-scale adaptive Wiener filtering. The decomposition result of $x_{1}(t)$ in Equation (6) by VMD is shown in Figure 3. The curve has obvious disturbances, and the sudden information loss of the square wave signal is more serious.

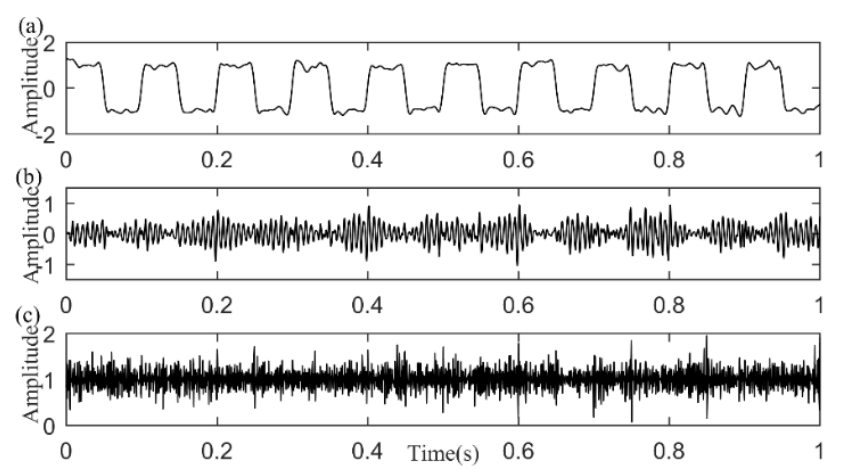

Figure 3. Decomposition results of VMD for $x_{1}(t)$. (a) Square wave components corresponding to IMF; (b) IMF corresponding to the AM-FM sine component; (c) Noise component.

\subsection{EEMD Algorithm}

EMD uses an interpolation algorithm to iteratively extract the mean value of the upper and lower envelopes and decompose the original signal into several IMFs.

(1) Set $h_{i}(t)=x(t), j=1$ and $i=1$.

(2) Use the cubic spline function to interpolate the poles of $h_{i}(t)$ to obtain the lower envelope $m_{l}(t)$ and the upper envelope $m_{u}(t)$, and then update $h_{i}(t)$ :

$$
h_{i+1}(t)=h_{i}(t)-\frac{m_{u}(t)+m_{i}(t)}{2}
$$

(3) Repeat step 2 until $h_{i+1}(t)$ meets the requirements defined by IMF; then, extract the $j$-th component:

$$
I M F_{j}(t)=h_{i+1}(t)
$$

(4) Set $r(t)=x(t)-I M F_{j}(t)$, update $x(t)$ with the remaining $r(t)$, and then repeat the previous three steps until $r(t)$ becomes a monotone function. The decomposition result can be expressed in the following form:

$$
x(t)=\sum_{j=1}^{N} I M F_{j}(t)+r(t)
$$

In non-stationary signal processing, the EMD algorithm shows good performance. However, mode mixing is a major shortcoming of EMD; if the analyzed signal is a strong noise signal, this shortcoming may cause severe errors. Therefore, in order to improve the ability to resist mode mixing and noise interference, the EEMD algorithm is proposed. In contrast, the difference is that the EEMD algorithm averages the decomposition results and adds different levels of white noise to the original signal. After the averaging process of the EEMD algorithm, part of the noise in the original signal will be canceled out because the added white noise signals are uncorrelated. 
The essence of the methods that are not based on the Fourier transform is to obtain the envelope of the extreme points by using interpolation functions to generate smooth envelopes such as the EMD algorithm and the EEMD algorithm. When dealing with broadband components, the final decomposition results will also become smooth because the IMFs are obtained by calculating the envelopes. The decomposed results corresponding to $x_{1}(t)$ in Equation (6) by applying EEMD are shown in Figure 4. It can be clearly seen that the IMF component obtained by EEMD is close to a sinusoidal signal; it is evident that the characteristics of the extracted square wave signal have changed.

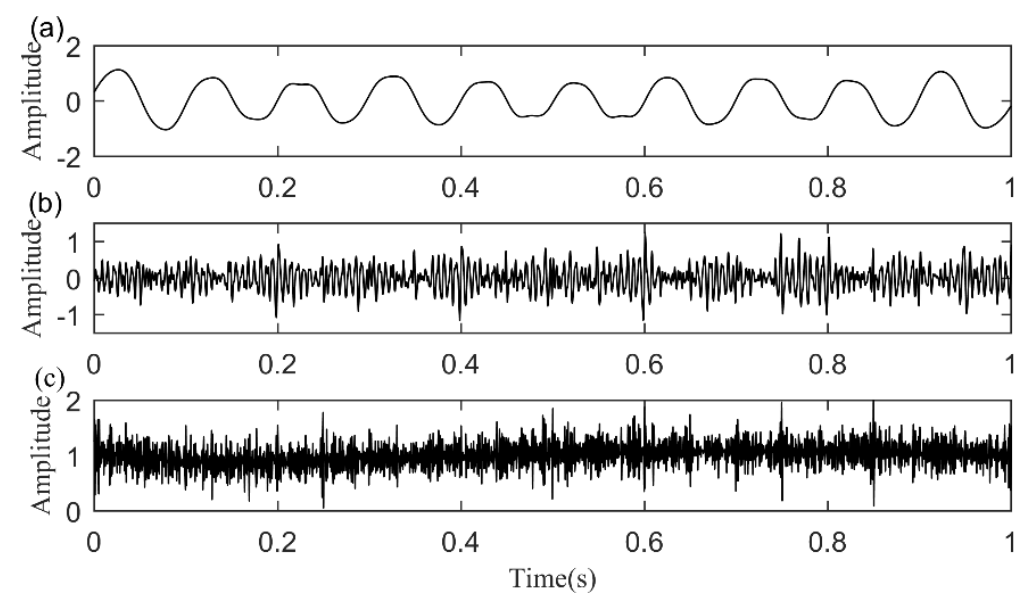

Figure 4. Decomposition results $x_{1}(t)$ by EEMD. (a) Square wave components corresponding to IMF; (b) IMF corresponding to the AM-FM sine component. (c) Noise component.

\section{MBMD Algorithm}

\subsection{Narrowband Signal}

The definition of the narrowband signal is shown in Equation (13):

$$
x_{\text {narrow }}(t)=A(t) \cos (\omega t+\phi(t))
$$

where $\phi(t)$ is function, and this function changes slowly. $A(t)$ is limited by the frequency band, $\omega^{\prime}$ is its central frequency, which is far less than $\omega$. The relative bandwidth is $2 \omega^{\prime} / \omega$, and it is far lower than 1.

In Equation (13), the regulated difference operator of the BMD algorithm can effectively distinguish narrowband signals from noise because wideband or narrowband signals are always smoother than noise, and the operator $D^{(2)}$ can represent the smoothness of nonstationary signals. To constrain the generated components in order for them to be smoother than the original signal, the regulated differential operator is constructed in the BMD algorithm. The operator can almost be applied to broadband signals, although it is suitable for narrowband signals.

\subsection{Broadband Signal}

For broadband signals, the Fourier series of sawtooth signals is as follows, while the Fourier series of square wave signals is given by Equation (5).

$$
\operatorname{sawtooth}(t)=\sum_{i=1}^{+\infty} \frac{(-1)^{(i-1)}}{(2 i-1)^{2}} \sin [(2 i-1) t]
$$


According to Equations (5) and (14), as the frequency increases, the magnitude of the sinusoidal component decreases. Therefore, the general form of the broadband signals can be constructed as follows:

$$
x_{\text {broad }}(t)=\sum_{i=1}^{+\infty} A_{i} \sin \left[i \omega t+\theta_{i}(t)\right]
$$

when $\omega \rightarrow \infty, A \rightarrow 0$, and $A_{i}$ decrease along with the increasement of $\omega, \theta(t)$ changes slowly.

The frequency of the wideband signal is a multiple of the narrowband signal shown in Equation (15), but even when the frequency amplitude decreases rapidly, the broadband signal can be distinguished from the noise because the wideband signal is still smoother than the noise signal. However, because the modulation differential operator was originally designed for narrowband signals, there are problems with broadband signals. For $x_{\text {narrow }}(t)$, the first Fourier series $A \cos (\omega t+\phi(t))$ multiplied by an envelope $A(t) / A$ should be its theoretically generated component. As for the narrowband signal, there is only one dominant frequency $\omega$.

However, there are multiple principal frequencies in $x_{\text {broad }}(t)$ which have different amplitudes such as $A_{i-1} \sin \left[(i+1) \omega t+\theta_{i-1}(t)\right]$ and $A_{i} \sin \left[i \omega t+\theta_{i}(t)\right]$. Among the sinusoidal components, the frequency interval is $\omega$, but the center frequency of the broadband signal is also $\omega$. The definition of relative bandwidth is the value obtained by dividing the bandwidth by the center frequency, which is $\omega / \omega=1$. In Section 4.1 , the relative bandwidth of narrowband signals has been proven to be much less than 1 . The BMD algorithm may regard $A_{i} \sin \left[i \omega t+\theta_{i}(t)\right]$ and $A_{i-1} \sin \left[(i-1) \omega t+\theta_{i-1}(t)\right]$ as two different components because the relative bandwidth is not small enough, and then the algorithm may divide the broadband signal into several narrowband components when applied to a broadband signal with a strong noise interference.

\subsection{Modulated Differential Operator for Broadband Signals}

First, the broadband component is multiplied by the high-frequency sinusoidal signal as follows:

$$
x_{\text {broad }}^{\prime}(t)=x_{\text {broad }}(t) \sin \left(\omega^{\prime} t\right)=\sum_{i=1}^{+\infty} A_{i} \sin \left[i \omega t+\theta_{i}(t)\right] \sin \left(\omega^{\prime} t\right)
$$

where $\sin \left(\omega^{\prime} t\right)$ is a high-frequency, single-frequency signal with $\omega^{\prime}=M \omega$, and $M$ is a preset positive integer, which can usually be set to $5-10 . x^{\prime}$ broad $(t)$ is a modulated signal, and it can then be converted to the following form:

$$
x_{\text {broad }}^{\prime}(t)=\frac{1}{2} \sum_{i=1}^{+\infty} A_{i} \cos \left[\left(\omega^{\prime}-i \omega\right) t-\theta_{i}(t)\right]+\frac{1}{2} \sum_{i=1}^{+\infty} A_{i} \cos \left[\left(\omega^{\prime}+i \omega\right) t+\theta_{i}(t)\right]
$$

The center frequency of the broadband signal is moved from $\omega$ to $\omega^{\prime}-\omega$ and $\omega^{\prime}+\omega$. The relative bandwidths are much lower than 1 , which are $\omega /\left(\omega^{\prime}-\omega\right)$ and $\omega /\left(\omega^{\prime}+\omega\right)$, respectively. After modulation, the relative bandwidth of a narrowband signal can be easily proved to also be much lower than 1 . Therefore, the modulation difference operator can be constructed as follows:

$$
T_{j}=\left\|D^{(2)}\left[I M F_{i}^{j}(t)\right]\right\|_{2}^{2}+\lambda\left\|D^{(2)}\left[x(t) \sin \left(\omega^{\prime} t\right)-I M F_{i}^{j}(t)\right]\right\|_{2}^{2}
$$

$\left\|D^{(2)}\left[I M F_{i}^{j}(t)\right]\right\|_{2}^{2}$ is minimized to ensure the smoothness of the decomposed IMFs, and $\left\|D^{(2)}\left[x(t) \sin \left(\omega^{\prime} t\right)-I M F_{i}^{j}(t)\right]\right\|_{2}^{2}$ is minimized to rapidly reduce the smoothness of the residual to obtain the sparsest decomposition result of the original signal. 


\subsection{MBMD Algorithm}

The main process of MBMD algorithm is outlined below, which is based on the modulation difference operator constructed in Equation (18). Figure 5 shows the flow chart of MBMD obtaining IMF components.

(1) $r_{0}(t)$ is set to equal $x(t)$.

(2) Separate direct current signals from the original signal.

$$
\begin{aligned}
& I M F_{0}(t)=\text { ifft }\left[\hat{r}_{0}(1)\right] \\
& r_{1}(t)=x(t)-I M F_{0}(t)
\end{aligned}
$$

where $i$ is set to equal $1, \hat{r}_{0}(k)$ is the FT of $r_{0}(t)$, ifft $\left[\hat{r}_{0}(1)\right]$ is the IFT of $\hat{r}_{0}(1)$.

(3) Construct the optimal problem $P 1$ as follows:

$$
\begin{array}{r}
\text { P1: Minimize } T_{1}\left(A_{1}, \omega_{1}, \theta_{1}, D_{1}\right), T_{2}\left(A_{2}, \omega_{2}, \theta_{2}, D_{2}\right), T_{3}\left[A_{3}(t), \omega_{3}(t), \theta_{3}(t)\right] \\
\text { S. T. } x(t)=\sum_{i=0}^{N} I M F_{i}(t)+\operatorname{res}(t), I M F_{i}^{j} \in D i c_{j} \\
T_{j}=\left\|D^{(2)}\left[I M F_{i}^{j}(t)\right]\right\|_{2}^{2}+\lambda\left\|D^{(2)}\left[x(t) \sin \left(\omega^{\prime} t\right)-I M F_{i}^{j}(t)\right]\right\|_{2}^{2}
\end{array}
$$

where $D^{(2)}$ is the quadratic differential form operator, $T_{j}$ is the modulated differential operator defined in Equation (18), $\lambda>0$ is always set to equal 1. $\omega^{\prime}=M \omega, \omega$ equals the maximum value for the frequency of $r_{i}(n)$. M can be generally set to 5-10.

(4) Use ACROA to solve $L 1$ to obtain the optimal value $T_{j(j=1,2,3)}$, find the minimum $T_{j}$ and choose the optimal $I M F_{i}(t)$ of $T_{j}$. Figure 5 illustrates the flow of this method.

(5) Update $r_{i}(t): r_{i+1}(t)=r_{i}(t)-I M F_{i}(t)$ after getting the best IMF.

(6) Terminate the iteration process if the termination condition of Equation (23) is met; otherwise, return to step 3 and continue to separate the signal until the termination condition is met.

$$
\left\|I M F_{i}(t)-I M F_{i-1}(t)\right\|_{2}^{2} /\left\|I M F_{i-1}(t)\right\|_{2}^{2} \leq \varepsilon
$$

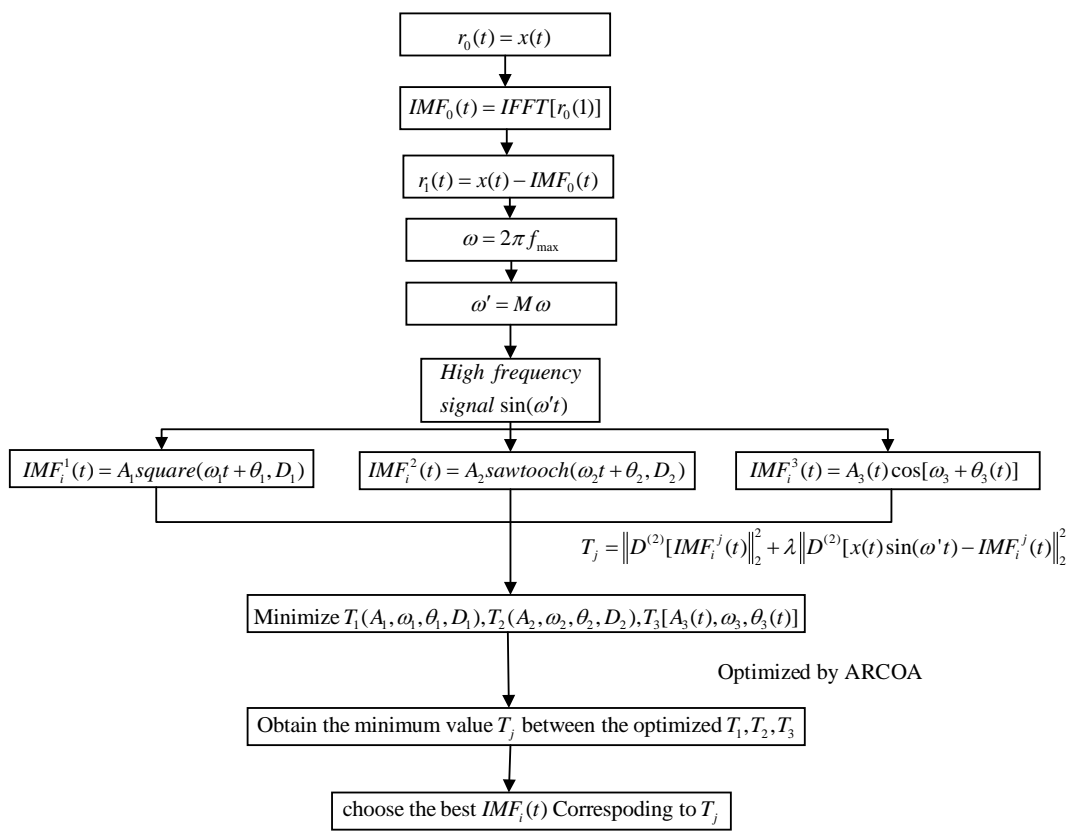

Figure 5. Flow chart of MBMD to obtain IMF. 
The MBMD method is used to decompose the photovoltaic DC current signal, while the DC signal, pulse signal, distortion and harmonic signals and the noise signal are separated and extracted.

\section{Introduction of Other Algorithms CMFE Algorithm}

In order to alleviate the shortening of the time series in the multi-scale fuzzy entropy (MFE) coarse-grained time series, CMFE applies the average MFE of different coarsegrained time series, which has the same proportion of factors as the extracted features. If the CMFE of a given signal is larger, it means that the given signal is more complex and contains more pattern information. The smaller the fluctuation range of the CMFE, the better the decomposition effect of an algorithm is and the more accurate the extracted signal features become. The calculation process of the CMFE algorithm is as follows:

(1) Calculate a different coarse-grained time series $y_{k}^{(\tau)}=\left\{y_{k, 1}^{(\tau)}, y_{k, 2}^{(\tau)}, K, y_{k, j}^{(\tau)}\right\}$ with a scale factor $\tau$, for a given signal $x(n)$ with $\mathrm{N}$ data points.

$$
y_{k, j}^{(\tau)}=\frac{1}{\tau} \sum_{i=(j-1) \tau+k}^{j \tau+k-1} x_{i}, 1 \leq j \leq N / \tau, 1 \leq k \leq \tau
$$

(2) Calculate the fuzzy entropy of each coarse-grained time series $y_{k}^{(\tau)}$, and then obtain the CMFE with the scale factor $\tau$ by averaging all the fuzzy entropies.

$$
\operatorname{CMFE}(x(n), \tau)=\frac{1}{\tau} \sum_{k=1}^{\tau} \operatorname{FuzzyEn}\left(y_{k}^{(\tau)}\right)
$$

\section{Simulation Analysis}

The simulation signal of a photovoltaic DC current disturbance is established, as shown in Figure 6. The second harmonic with an amplitude of $0.02 \mathrm{~A}$ and a frequency of $100 \mathrm{~Hz}$ is set in the signal, where disturbance $A$ is the disturbance generated by linear load access or connection; disturbance $B$ is the disturbance caused by nonlinear load access or connection; disturbance $C$ is the current pulse disturbance, and $n_{1}(t)$ is white noise with an SNR of 25. The signal model formula is as follows:

$$
\begin{gathered}
f(t)=2+0.02 \cos (200 \pi t)+A+B+B 1\left[-2 \times(t-2.5)^{2}+0.5\right]+B 2\left[2 \times(t-4)^{2}\right] \\
+B 3\left[2 \times(t-5.5)^{2}-0.5\right]+B 4\left[-2 \times(t-7)^{2}\right]+C \sin (50 \pi t)+n_{1}(t) \\
A=\left\{\begin{array}{cc}
2 & 0.2 \leq t \leq 0.7 \\
-1.5 & 1.0 \leq t \leq 1.7 \\
0 & t=\text { else }
\end{array}, B=\left\{\begin{array}{cc}
0.5 & 2.5 \leq t \leq 3.5 \\
-0.5 & 5.5 \leq t \leq 6.5 \\
0 & t=\text { else }
\end{array}\right.\right. \\
B 3=\left\{\begin{array}{ccc}
1 & 5 \leq t \leq 5.5 \\
0 & t=\text { else }
\end{array}, B 4=\left\{\begin{array}{cc}
1 & 6.5 \leq t \leq 7 \\
0 & t=\text { else }
\end{array}, C=\left\{\begin{array}{cc}
2.5 & 9 \leq t \leq 9.2 \\
0 & t=\text { else }
\end{array}\right.\right.\right.
\end{gathered}
$$

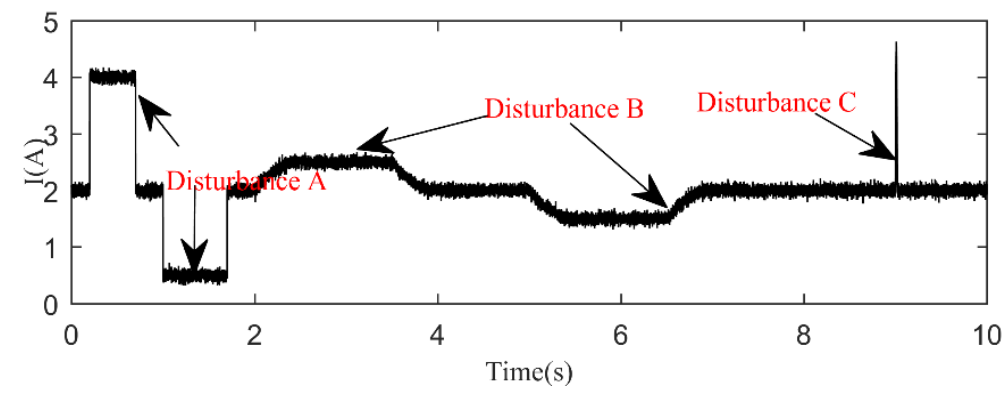

Figure 6. Photovoltaic DC simulation signal diagram. 
When analyzing broadband signals, the VMD algorithm is still affected by the Gibbs phenomenon because its essence is the multi-scale adaptive Wiener filtering. Figure 7 shows the decomposition results of the photovoltaic DC simulation electrical signals by VMD.
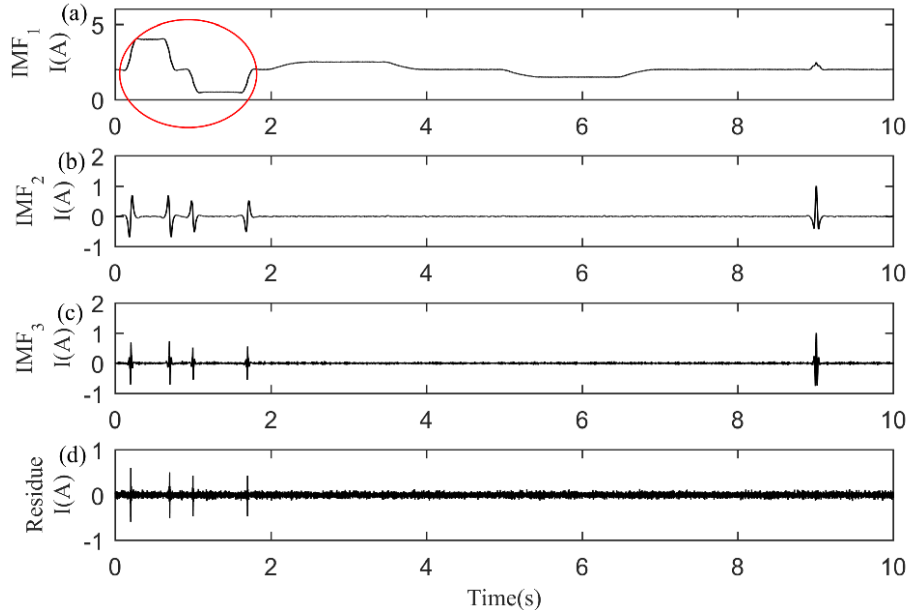

Figure 7. VMD simulation signal decomposition results. (a) $I M F_{1}$ component; (b) $I M F_{2}$ component; (c) $I M F_{3}$ component; (d) Residue.

It can be seen in Figure 7a that the mutation information of the square wave signal in the $I M F_{1}$ component decomposed by VMD is lost, the Gibbs phenomenon appears, and the missing characteristic information appears separately in the remaining IMF components. Figure $7 \mathrm{~d}$ is the residual component.

To obtain the envelope of the extreme points, the EMD method and the EEMD method typically use interpolation functions, which are non-Fourier transform-based methods, thereby generating a smooth envelope. When dealing with the broadband component, since the IMF is obtained by calculating the envelope, the final decomposition results are also smoothed. Figure 8 shows the decomposition result of the photovoltaic current simulation signal using EEMD. It can be seen that the obtained $I M F_{1}$ and $I M F_{2}$ are components with partial signal characteristics, the $I M F_{3}$ component is close to a sinusoidal signal, and $I M F_{4}$ is close to the DC component. Evidently, the extracted signal characteristics have changed.
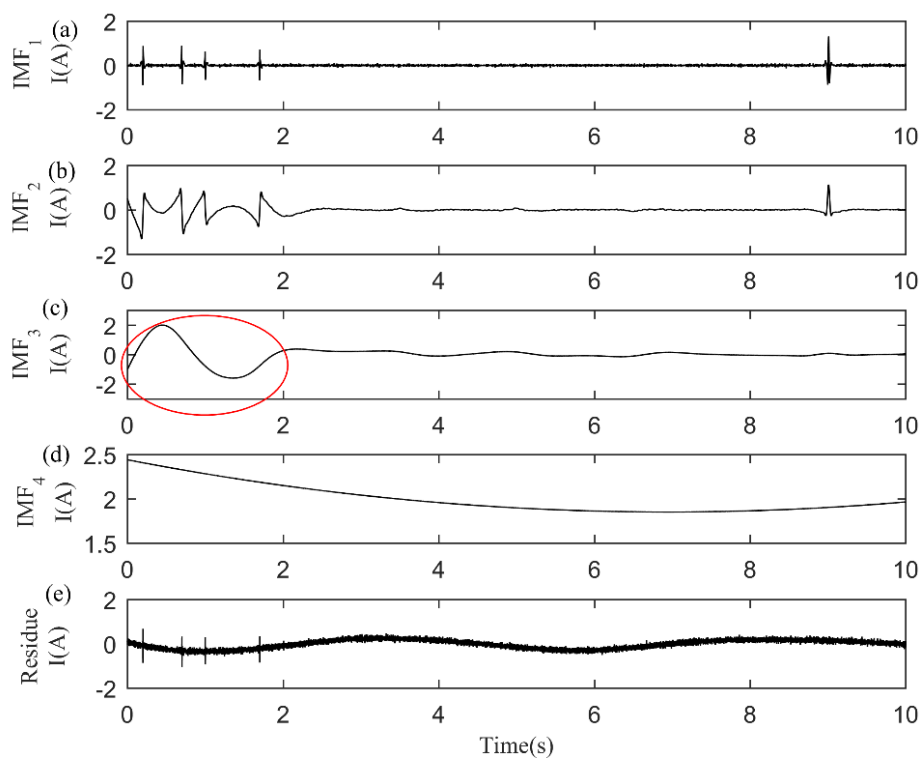

Figure 8. EEMD simulation signal decomposition results. (a) $I M F_{1}$ component; (b) $I M F_{2}$ component; (c) $I M F_{3}$ component; (d) $I M F_{4}$ component; (e) Residue. 
It can be seen in Figure 9 that the MBMD method is used to decompose the DC current signal, which can separate and extract the DC signal, pulse signal, distortion and harmonic signals, and the noise signal.
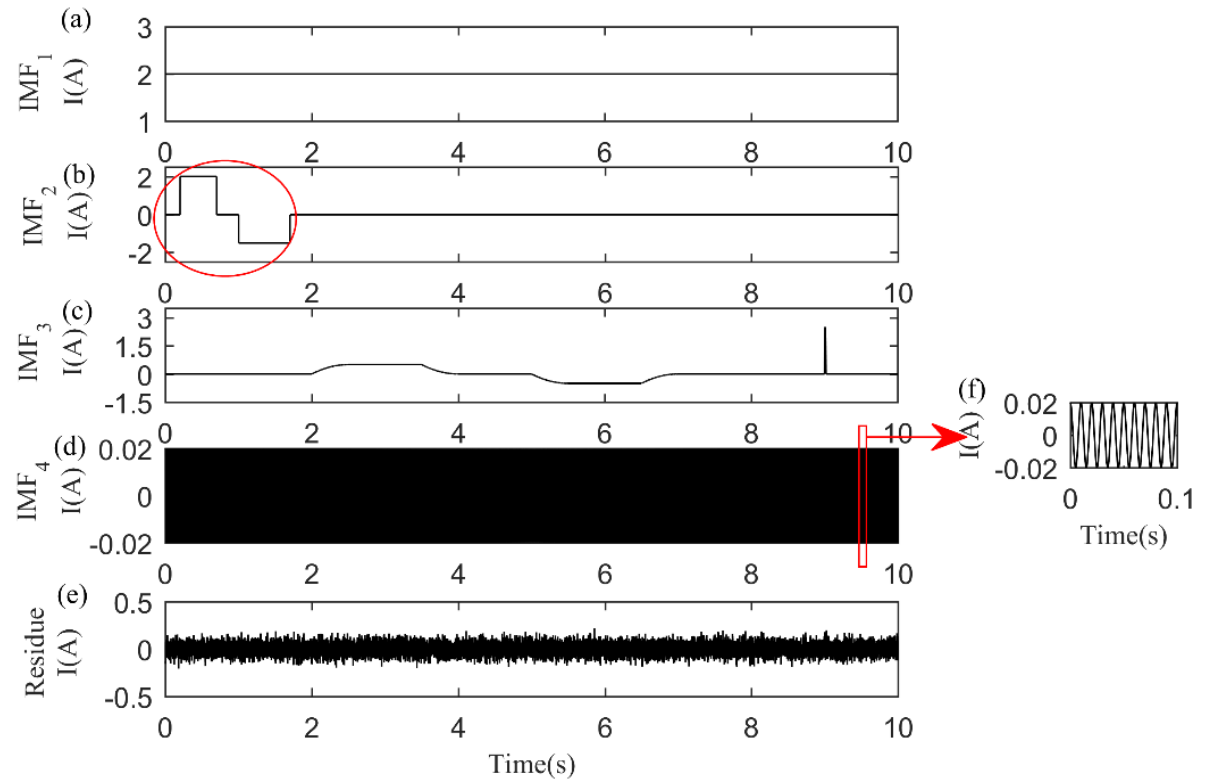

Figure 9. Simulation signal decomposition diagram of MBMD. (a) DC signal; (b) Wideband signal; (c) Narrowband signal; (d) Harmonic signal; (e) Noise signal; (f) A 0.1 s signal extract from (d).

Table 1 lists the accuracy parameters of the separated IMF obtained by the three algorithms, including the accuracy parameters of the correlation coefficient $r_{1}$, the energy error $E_{1}$, and the time $T$ to make a more accurate comparison.

Table 1. Evaluation parameters of the three algorithms (SNR 25).

\begin{tabular}{cccc}
\hline Method & $\boldsymbol{r}_{\mathbf{1}}$ & $\boldsymbol{E}_{\mathbf{1}}$ & $\boldsymbol{T}(\mathbf{s})$ \\
\hline VMD & 0.9543 & 0.0159 & 4.7134 \\
EEMD & 0.8052 & 0.5835 & 9.3256 \\
MBMD & 0.9981 & 0.0015 & 34.3517 \\
\hline
\end{tabular}

In order to compare the effect of signal decomposition under different signal-to-noise ratios, two additional simulation models are constructed here. Except for noise, the other components remain unchanged. White noise $n_{2}(t)$ with an SNR of 10 and white noise $n_{3}(t)$ with an SNR of 5 were added, respectively. The two signal models constructed are shown in Figures 10 and 11.

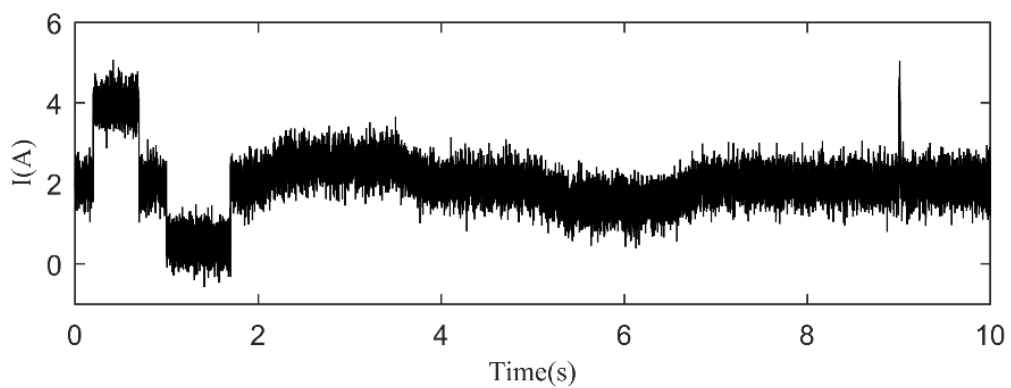

Figure 10. Photovoltaic signal simulation diagram under the noise of SNR 10. 


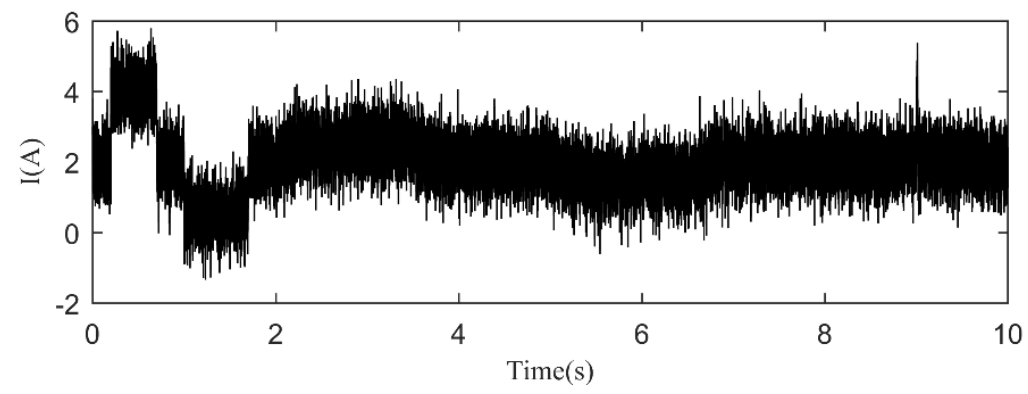

Figure 11. Photovoltaic signal simulation diagram under the noise of SNR 5.

Figures 12 and 13 show the VMD decomposition results of these two signal models. It can be seen that with the decrease of SNR, the components of the VMD containing noise increase, especially $I M F_{2}$ and $I M F_{3}$. Due to the increase of noise, the decomposed components also contain part of the noise signals, so that the characteristics of the square wave signal are not obvious. Figures 14 and 15 are the decomposition results of EEMD. Some components obtained by EEMD are relatively smooth, and the increased noise components are reflected in $I M F_{1}$. Figures 16 and 17 shows the decomposition results of MBMD. Tables 2 and 3 show the relevant parameters (energy error $E_{1}$, correlation coefficient $r_{1}$, and time T), respectively. It can be seen that the decomposition effect of MBMD is the best under different SNRs.
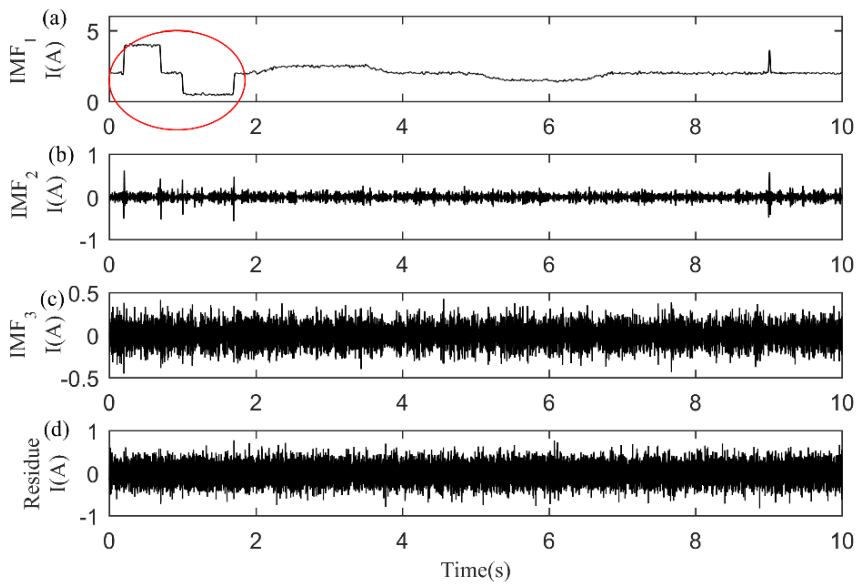

Figure 12. VMD decomposition diagram under the noise of SNR 10. (a) $I M F_{1}$ component; (b) $I M F_{2}$ component; (c) $I M F_{3}$ component; (d) Residue.
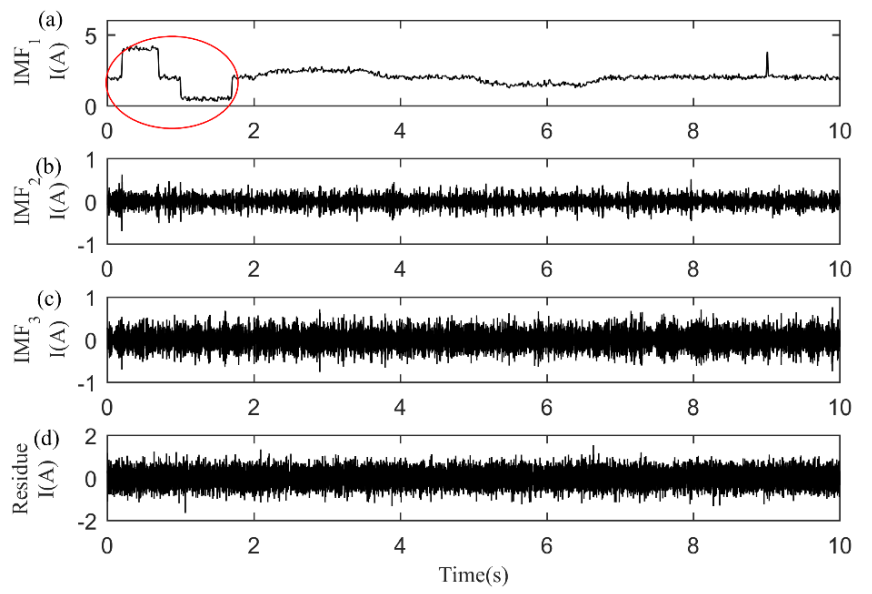

Figure 13. VMD decomposition diagram under the noise of SNR 5. (a) $I M F_{1}$ component; (b) $I M F_{2}$ component; (c) $I M F_{3}$ component; (d) Residue. 

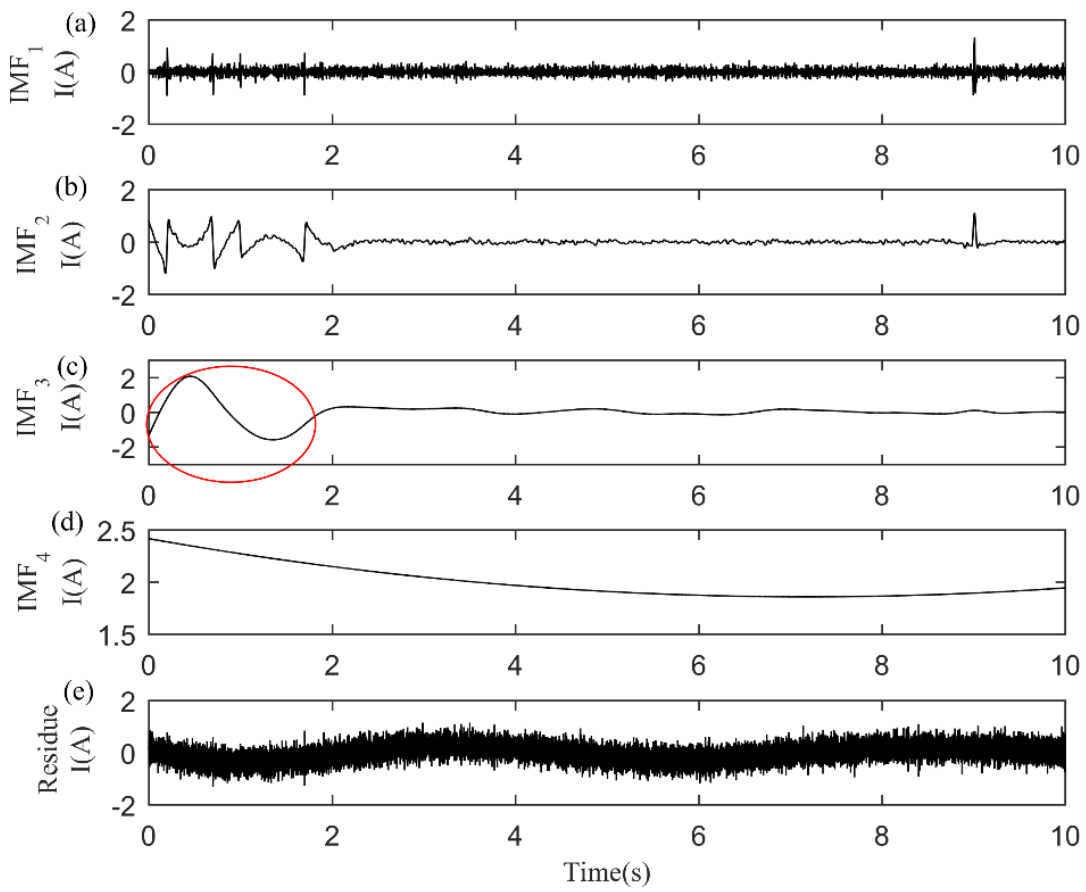

Figure 14. EEMD decomposition diagram under the noise of SNR 10. (a) $I M F_{1}$ component; (b) $I M F_{2}$ component; (c) $I M F_{3}$ component; (d) $I M F_{4}$ component; (e) Residue.
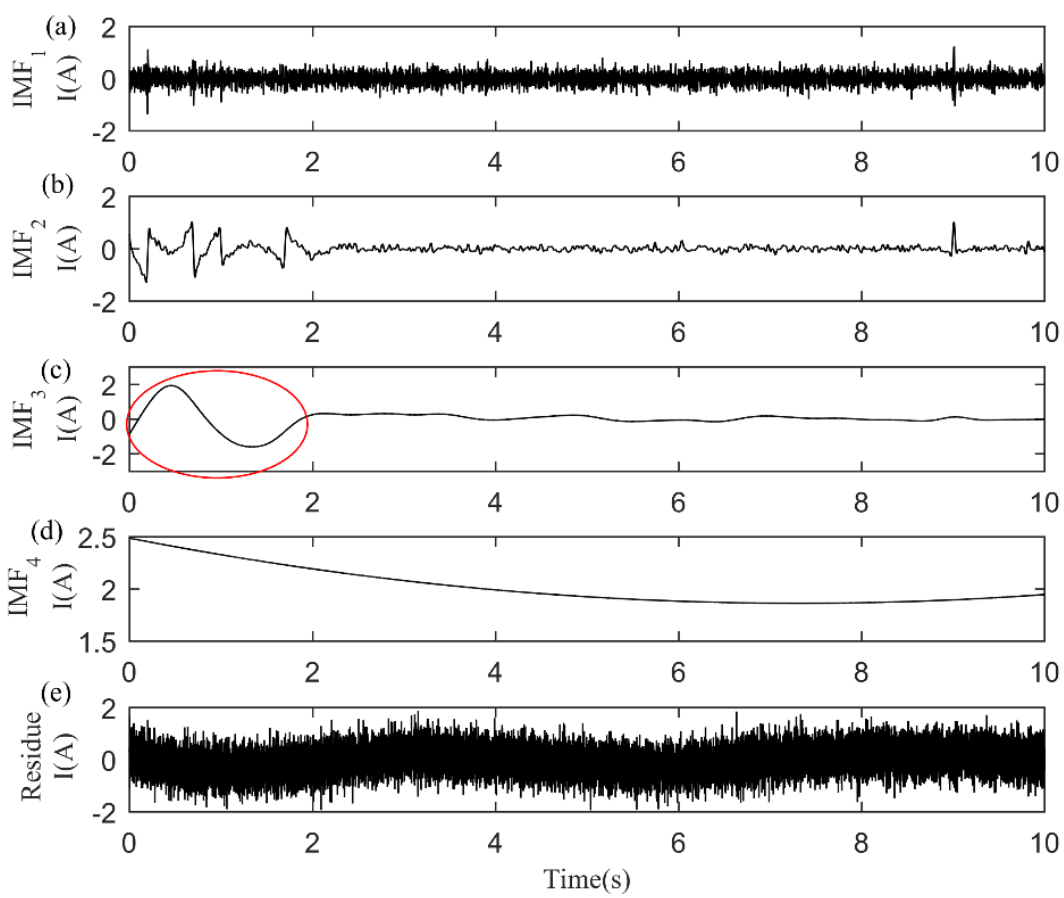

Figure 15. EEMD decomposition diagram under the noise of SNR 5. (a) $I M F_{1}$ component; (b) $I M F_{2}$ component; (c) $I M F_{3}$ component; (d) $I M F_{4}$ component; (e) Residue. 

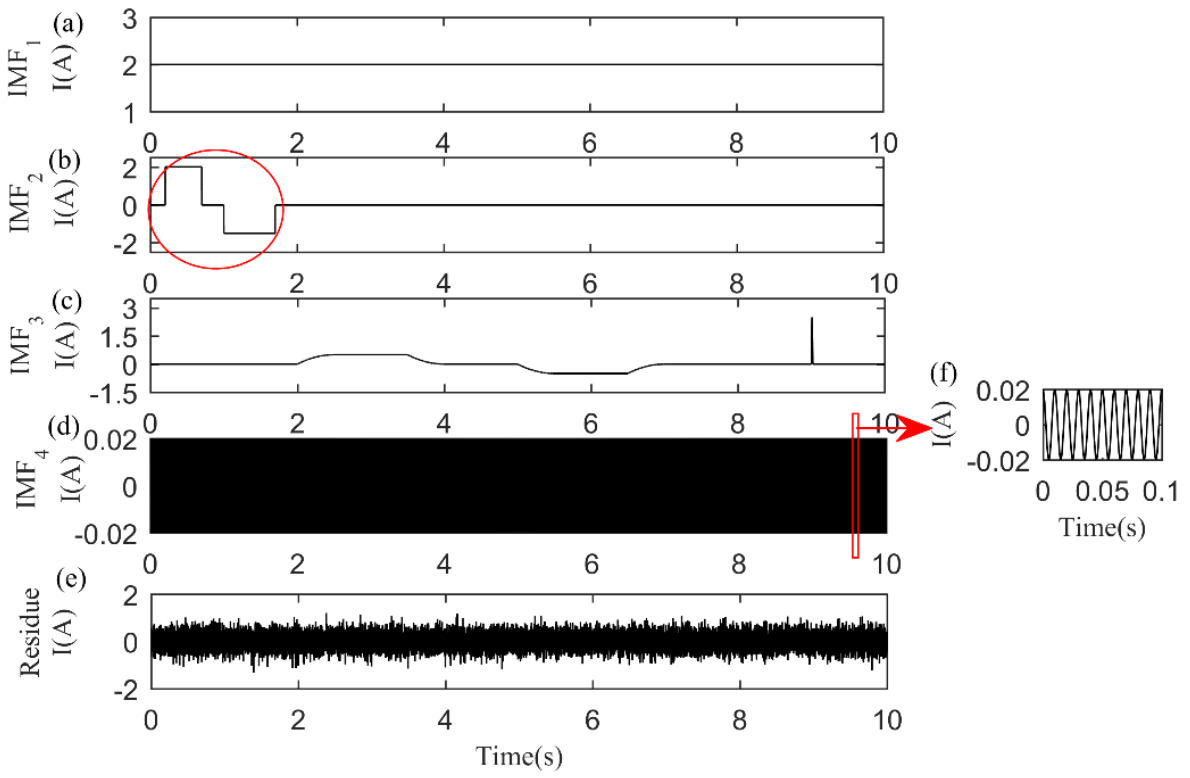

Figure 16. MBMD decomposition diagram under the noise of SNR 10. (a) DC signal; (b) Wideband signal; (c) Narrowband signal; (d) Harmonic signal; (e) Noise signal; (f) A 0.1 s signal extract from (d).
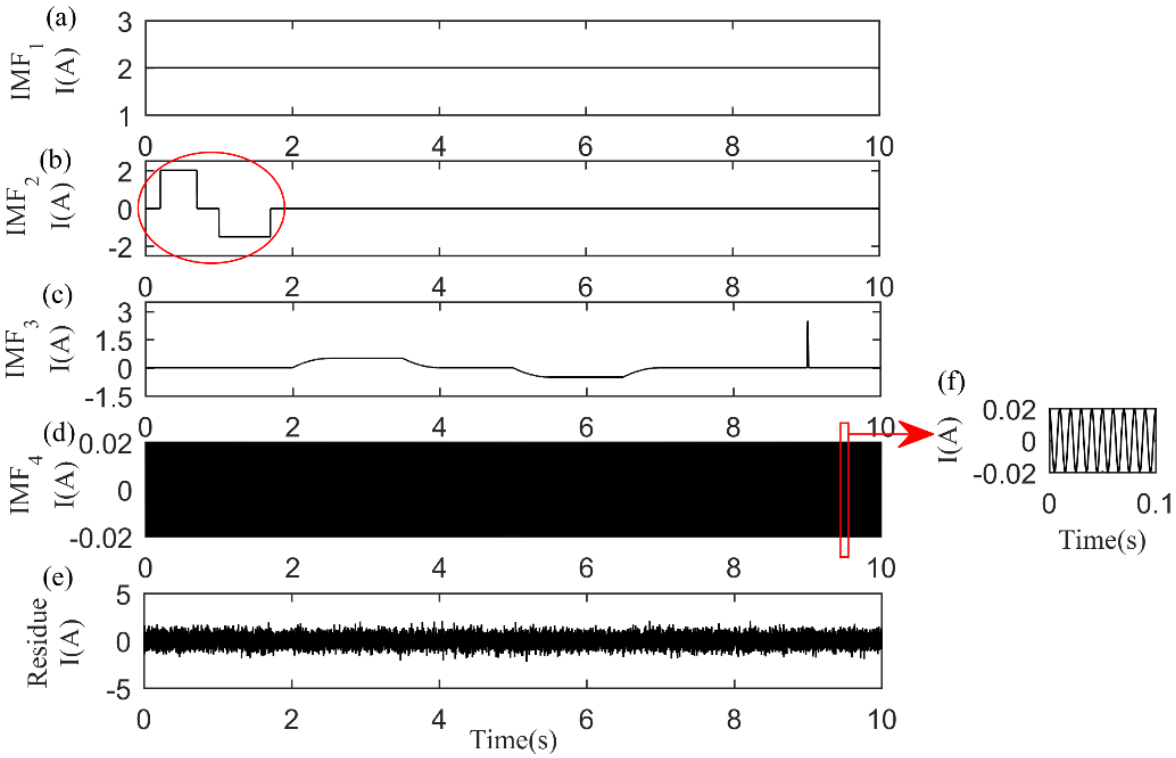

Figure 17. MBMD decomposition diagram under the noise of SNR 5. (a) DC signal; (b) Wideband signal; (c) Narrowband signal; (d) Harmonic signal; (e) Noise signal; (f) A 0.1 s signal extract from (d).

Table 2. Evaluation parameters of the three algorithms (SNR 10).

\begin{tabular}{cccc}
\hline Method & $\boldsymbol{r}_{\mathbf{1}}$ & $\boldsymbol{E}_{\mathbf{1}}$ & $\boldsymbol{T}(\mathbf{s})$ \\
\hline VMD & 0.9314 & 0.0314 & 4.9525 \\
EEMD & 0.7837 & 0.7493 & 9.7264 \\
MBMD & 0.9979 & 0.0017 & 35.5524 \\
\hline
\end{tabular}


Table 3. Evaluation parameters of the three algorithms (SNR 5).

\begin{tabular}{cccc}
\hline Method & $\boldsymbol{r}_{\mathbf{1}}$ & $\boldsymbol{E}_{\mathbf{1}}$ & $\boldsymbol{T}(\mathbf{s})$ \\
\hline VMD & 0.9197 & 0.0537 & 5.2497 \\
EEMD & 0.7529 & 0.8622 & 9.9633 \\
MBMD & 0.9977 & 0.0019 & 36.1146 \\
\hline
\end{tabular}

\section{Experimental Analysis}

The flow chart of experimental analysis is roughly shown in Figure 18:

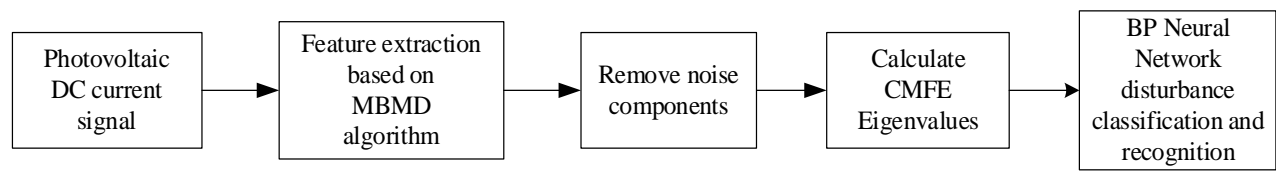

Figure 18. Flow chart of experimental analysis.

\subsection{Data Collection}

The experimental photovoltaic data were collected from the experimental platform for small photovoltaic measurement and the control system. As shown in Figure 19, the instrument used in the experiment has two $18 \mathrm{~V}, 50 \mathrm{~W}$ solar panels of $1480 \mathrm{~mm} \times 680 \mathrm{~mm}$, and the solar panels store electrical energy in batteries. The rated voltage of the battery as a stable power source is $12 \mathrm{~V}$. The controller of this photovoltaic system functions as a combiner box and a controller at the same time, which can control the charging and discharging of the battery and solar panels. The inverter parameters are $12 \mathrm{~V}, 1000 \mathrm{~W}$. The linear loads used are $36 \mathrm{~W}$ AC bulbs and $50 \mathrm{~W}$ DC bulbs. The nonlinear loads used are $8 \mathrm{~W}$ AC motors, $28 \mathrm{~W}$ AC motors, and 20.4 W DC motors. The signal measurement module contains four sensors; among them, the DC sensor uses a Hall sensor, while the rest are ordinary current and voltage sensors. These signals are collected by our self-made signal acquisition software, a specific collection software on the computer. The sampling time is 20 $\mathrm{s}$, and the signal acquisition frequency is $2 \mathrm{kHz}$. In the experiment, the switching sequence of each load was set first, and 50 sets of signals, including the no-load state, were obtained. After collecting the signals, the 15th set of signals was selected as the experimental signals.

\subsection{Experimental Analysis of Feature Extraction}

Select the 15th set of experimental signals. Figure 20 shows the signal collected through the photovoltaic experimental platform; for convenience of comparison, Figures 21a, 22a and 23a are all current signals collected in the experiment. It can be seen in Figure 20 that the transient rise and fall of the current in the original experimental signal caused by the switch of the DC bulb is similar to a regular rectangular signal, which is a broadband signal. Moreover, as the DC motors are turned off, two sets of current pulse signals are generated.

The 15th set of collected signals is decomposed by the VMD algorithm. It can be seen in Figure $21 \mathrm{~b}$ that after the current distortion generated by the DC load switch is decomposed, the mutation information of the Chinese wave signal of the $I M F_{1}$ component of the VMD decomposition is lost because of the Gibbs phenomenon; the mutation information and the current pulse signal are thus divided into a set as narrowband frequency signals.

The EEMD algorithm is used to decompose the collected 15th set of signals. It can be seen in Figure 22, among the IMF components obtained by the EEMD decomposition method, that $I M F_{3}$ is the closest to the original current signal. When processing broadband components, the final decomposition result will also become smooth because the IMF is obtained by calculating the envelope. 


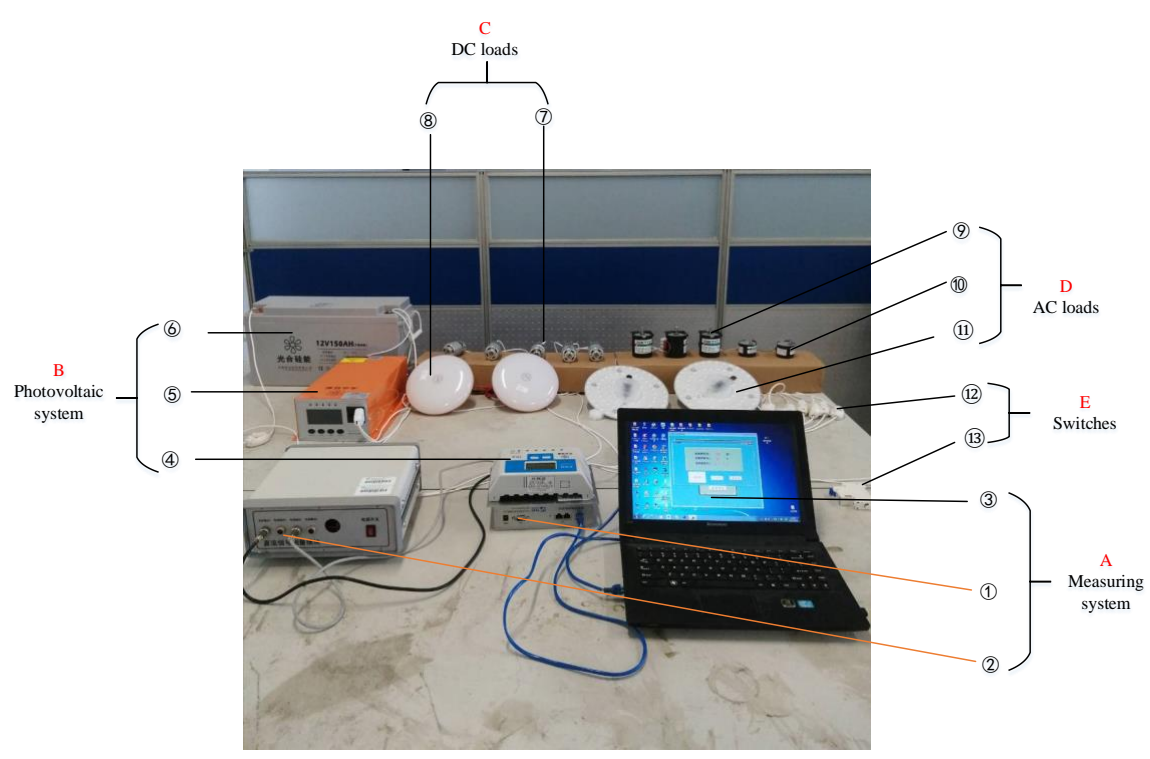

Figure 19. Experimental platform for small photovoltaic measurement and the control system. A: Measuring system: (1) Analog-to-digital conversion module; (2) Signal measurement module; (3) Computer terminal signal acquisition software; B: Photovoltaic system: (4) Photovoltaic system controller; (5) Inverter; (6) Storage battery (12 V); C: DC loads: (7) DC motor (20.4 W); (8) DC bulb (50 W); D: AC loads: (9) AC motor (28 W); (10) AC motor (8 W); (11) AC bulb (36 W); E: Switches: (12) Load switches; (13) Master switch.

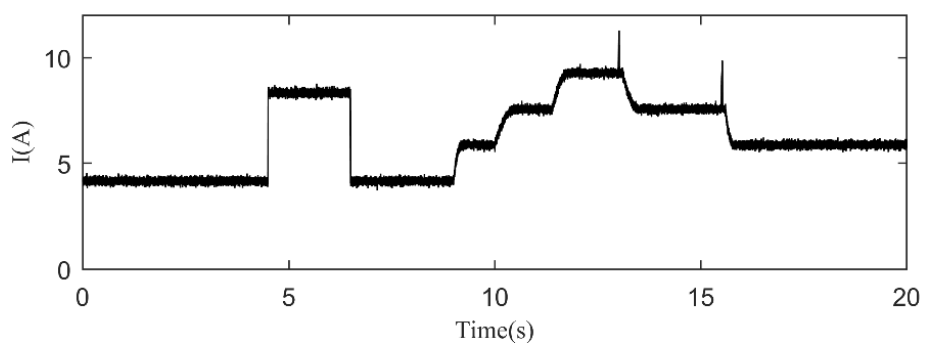

Figure 20. Experimental signal diagram.
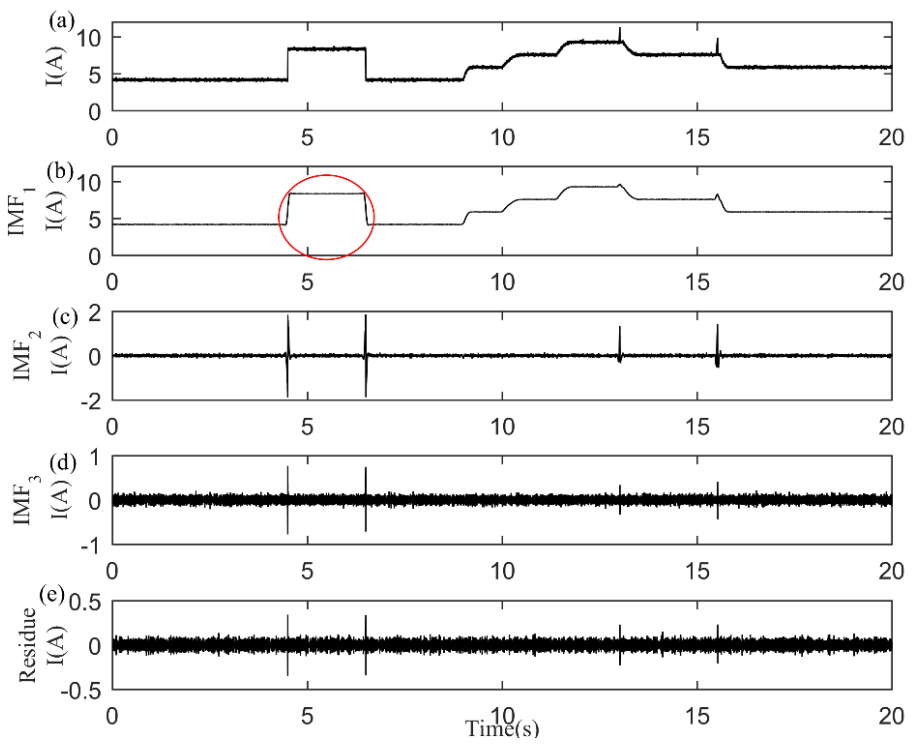

Figure 21. VMD decomposition diagram of the experimental signal. (a) Experimental current signal; (b) $I M F_{1}$ component; (c) $I M F_{2}$ component; (d) $I M F_{3}$ component; (e) Residue. 

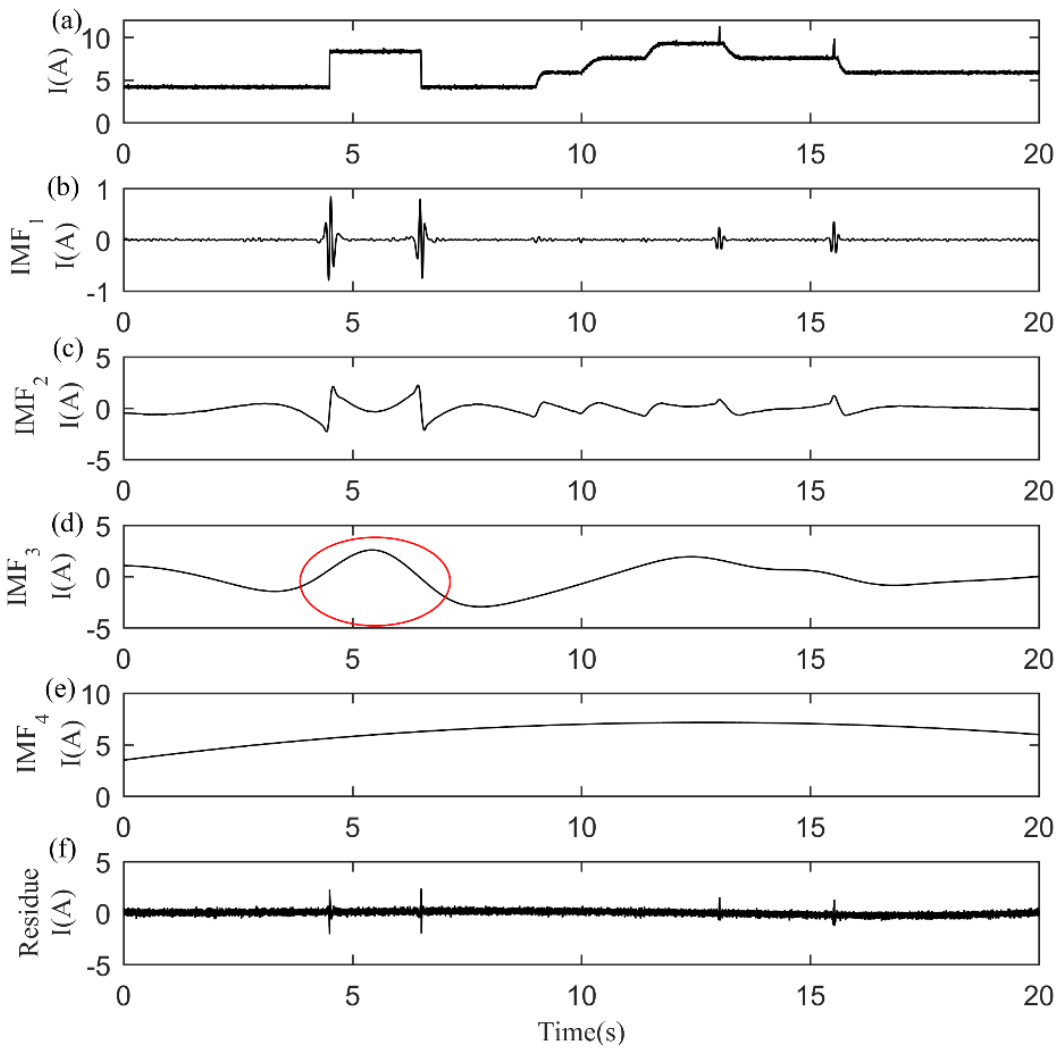

Figure 22. EEMD decomposition diagram of the experimental signal. (a) Experimental current signal; (b) $I M F_{1}$ component; (c) $I M F_{2}$ component; (d) $I M F_{3}$ component; (e) $I M F_{4}$ component; (f) Residue.

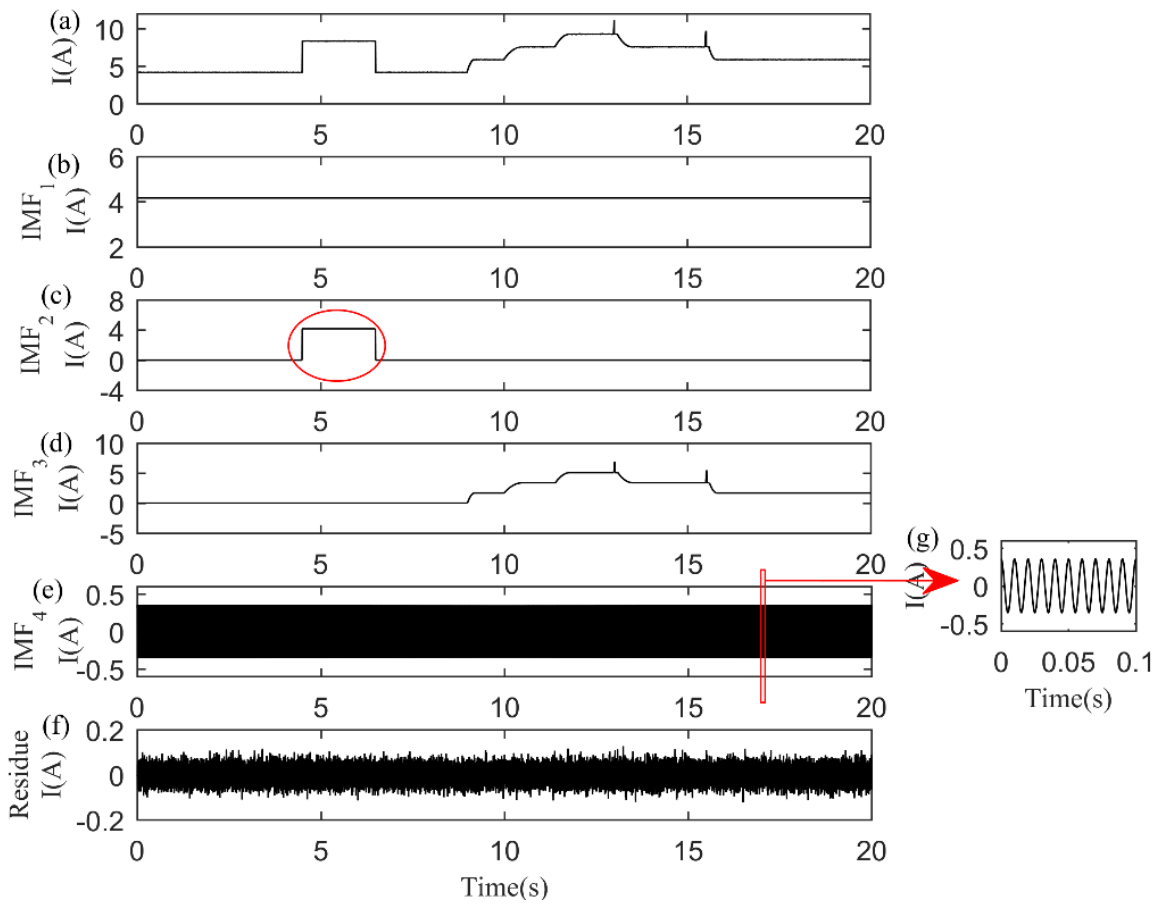

Figure 23. MBMD decomposition diagram of the experimental signal. (a) Experimental current signal; (b) DC signal; (c) Broadband signal; (d) Narrowband signal; (e) Harmonic signal; (f) Noise signal; (g) A $0.1 \mathrm{~s}$ signal extract from (d).

The MBMD algorithm was used to decompose the collected signals of set 15 , as shown in Figure 23. The MBMD algorithm divides the experimental signals into: Figure 23b, DC 
component; Figure 23c, broadband component; Figure 23d, current distortion component (narrowband signal); Figure 23e, second harmonic component; Figure 23f, harmonic and noise component. Since the second harmonics of the photovoltaic DC signal account for a large part of the signal, and the third harmonics and other high harmonics have very little content, the third harmonics and other high harmonics along with the noise components are divided together. It can be seen that the MBMD algorithm has a good decomposition effect on the feature information of the broadband signal, and is suitable for extracting effective features.

In order to compare the decomposition effect of the three algorithms of MBMD, VMD, and EEMD on the photovoltaic DC experimental signal more closely, CMFE was used to calculate the three algorithms. In addition, 40 sets of four common photovoltaic DC disturbance signals were collected in the experiment. The MBMD, VMD, and EEMD algorithms were used to decompose each disturbance, and the $I M F_{1}$ components obtained by each of the three algorithms were extracted by CMFE. To unify the standard, the scale factor of the composite multi-scale fuzzy entropy (CMFE) was set to 4, and a graph with a scale factor of 3 was used for comparison. The CMFE comparison chart of the three algorithms under the four disturbance states is presented in Figures 24-27.

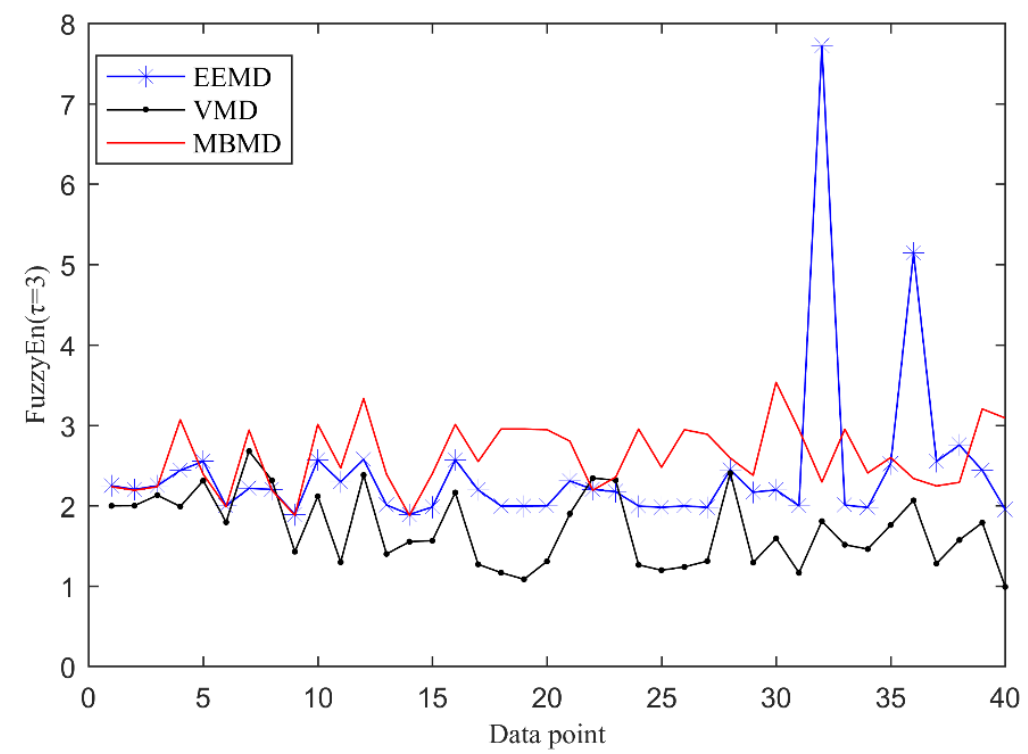

Figure 24. MBMD, VMD, EEMD comparison diagram of the harmonic signal.

Because harmonic disturbance always exists in the photovoltaic DC circuit, the disturbance states measured by the four experiments all contain harmonics. From the CMFE diagram above, it can be seen that among the various resolution methods, the MBMD method has the smallest CMFE fluctuation after decomposition, followed by VMD, and EEMD has the largest fluctuation; therefore, the MBMD decomposition has the best effect. In order to effectively and accurately distinguish the type of photovoltaic DC signal disturbance, the BP neural network algorithm was used to extract and classify the results after CMFE extraction.

\subsection{Disturbance Identification}

At this point, 40 sets of photovoltaic DC signal samples of the following four states were selected from the collected experimental signals. Because harmonics always exists in the photovoltaic DC circuit, and although it is also a kind of disturbance, it is marked as state 1; the other three states have a disturbance in addition to harmonics. State 1 is the harmonics; State 2 is the current distortion of the DC linear load plus harmonics; State 3 is the current distortion of the DC non-linear load plus harmonics; State 4 is the current pulse plus harmonics. 
Comparing the CMFE decomposed by MBMD from Figures 25 and 26, it can be seen that the magnitude of the CMFE decomposed by the two disturbance states is very close. In order to prevent the BP neural network algorithm from mixing these two types of signals into one state, the first three IMF components decomposed by different algorithms are calculated by CMFE, and the CMFE of the three IMF components is used as the standard to classify the disturbance state. To illustrate that the CMFE of the three IMF components can be classified, we used the MBMD decomposition algorithm as an example: We took 40 sets of sample signals for each state (160 sets of signals in total) and decomposed the signals in the four disturbance states in the samples. Subsequently, we took the first three IMF components obtained by decomposition, calculated the respective CMFE values, and also calculated the value range as well as the mean and range of the CMFE. Table 4 shows the CMFE values of the three IMF components obtained by MBMD decomposition. It can be seen that four states can be distinguished by comparing the values of $I M F_{1}, I M F_{2}$, and $I M F_{3}$.

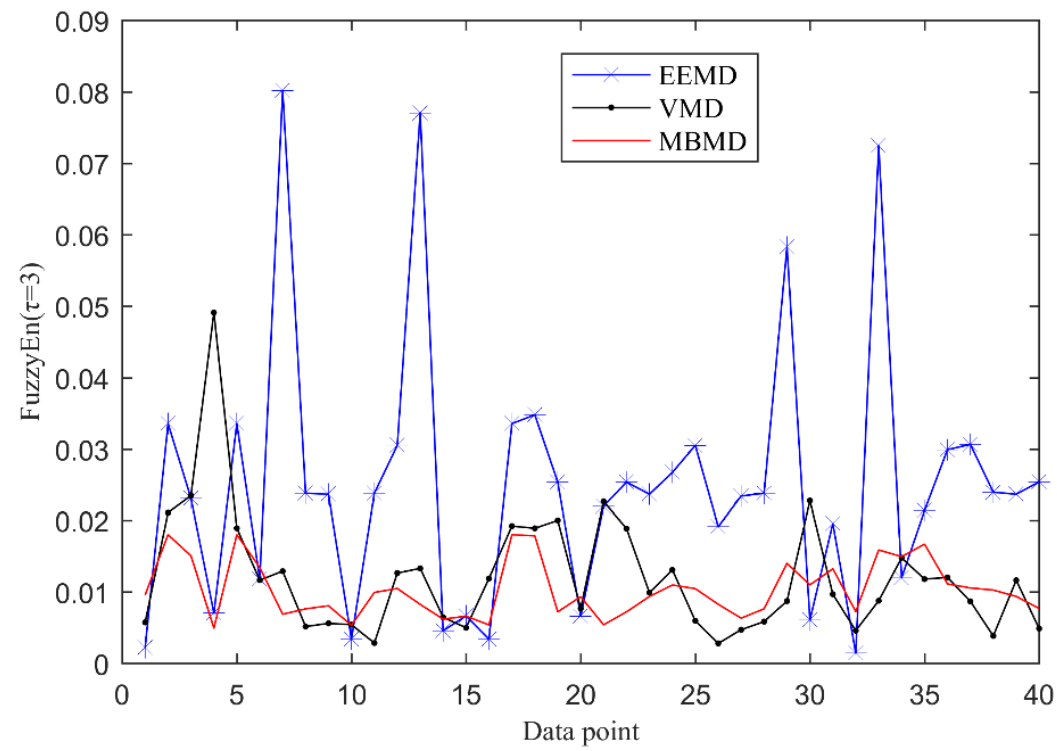

Figure 25. MBMD, VMD, EEMD comparison diagram of the harmonic signal plus the square wave signal.

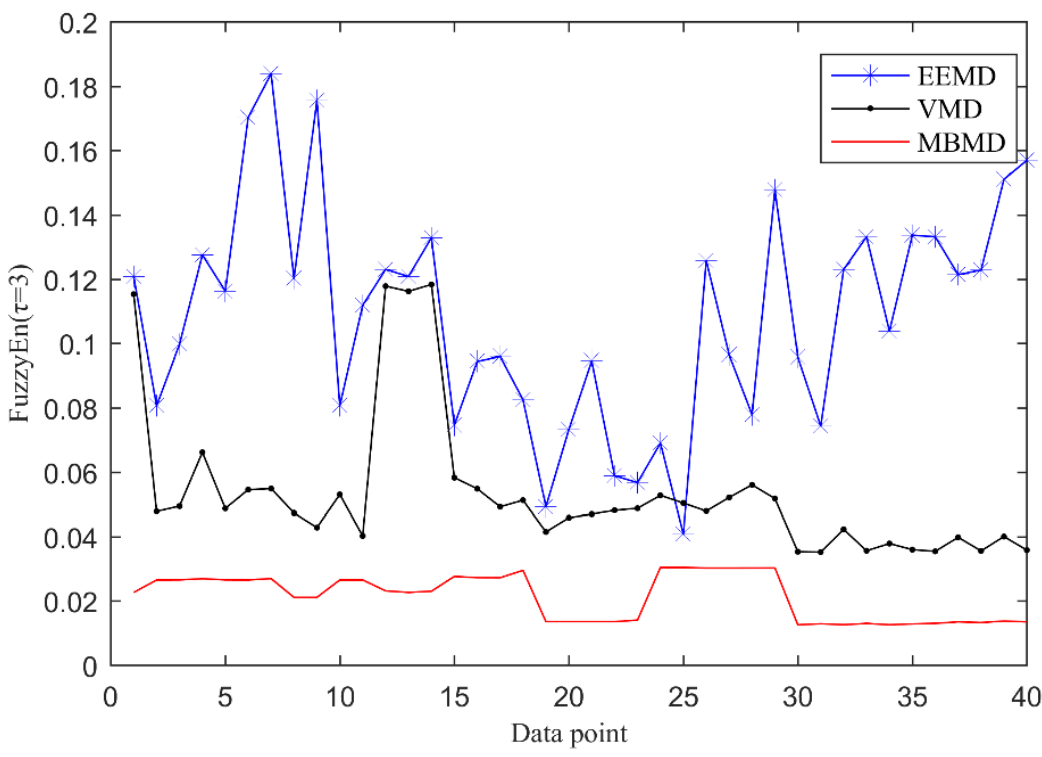

Figure 26. MBMD, VMD, EEMD comparison diagram of the harmonic signal plus the narrowband signal. 


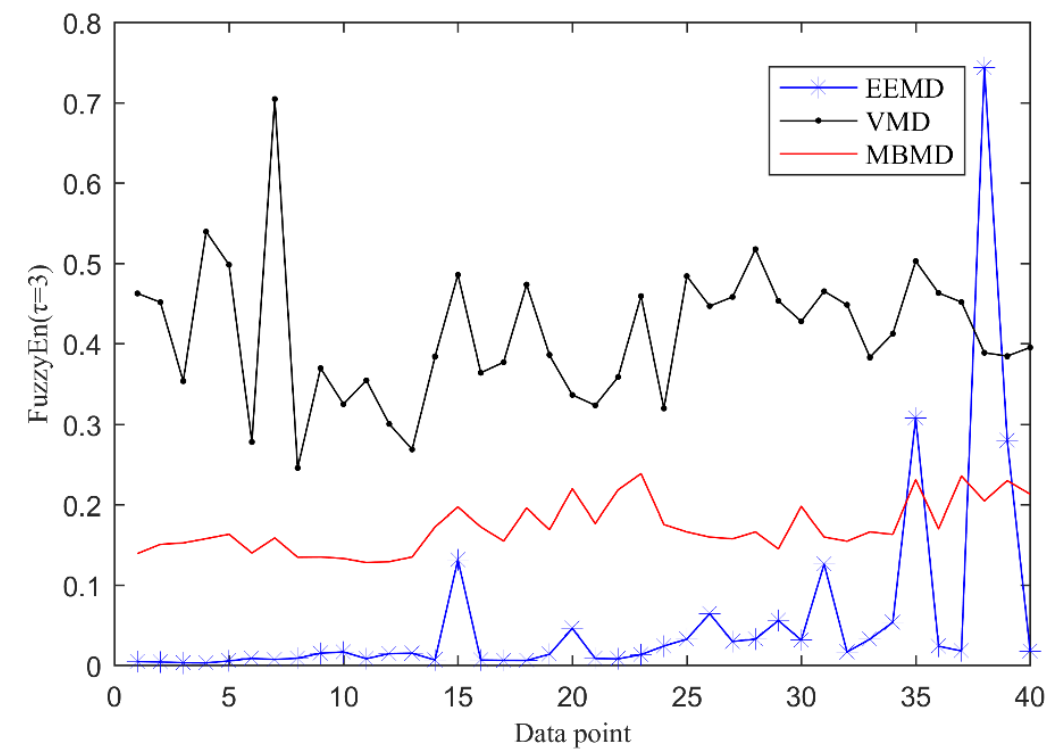

Figure 27. MBMD, VMD, EEMD comparison diagram of the harmonic signal plus the pulse signal.

Table 4. CMFE evaluation parameters of MBMD in four states.

\begin{tabular}{ccccc}
\hline \multirow{2}{*}{ State } & IMF & \multicolumn{3}{c}{ CMFE of MBMD } \\
\cline { 2 - 4 } & & Value Range & Mean Value & Variance \\
\hline \multirow{3}{*}{ State 1} & $I M F_{1}$ & $1.8781-3.5363$ & 2.6117 & 0.1686 \\
& $I M F_{2}$ & $0.5583-0.7261$ & 0.6484 & 0.0023 \\
& $I M F_{3}$ & $1.0725-1.2732$ & 1.1343 & 0.0027 \\
\hline \multirow{3}{*}{ State 2 } & $I M F_{1}$ & $0.0049-0.0180$ & 0.0103 & $1.5732 \times 10^{-5}$ \\
& $I M F_{2}$ & $0.0021-0.0043$ & 0.0107 & $5.5222 \times 10^{-5}$ \\
State 3 & $I M F_{3}$ & $0.1245-0.3233$ & 0.1744 & 0.0019 \\
& $I M F_{1}$ & $0.0126-0.0305$ & 0.0213 & $5.0052 \times 10^{-5}$ \\
& $I M F_{2}$ & $0.0311-0.2566$ & 0.0784 & 0.0040 \\
State 4 & $I M F_{3}$ & $1.1450-1.5470$ & 1.2687 & 0.0109 \\
& $I M F_{1}$ & $0.1281-0.2385$ & 0.1717 & 0.0010 \\
& $I M F_{2}$ & $0.3203-0.6647$ & 0.5849 & 0.0068 \\
& $I M F_{3}$ & $0.0598-0.5988$ & 0.1430 & 0.0078 \\
\hline
\end{tabular}

According to the data in Table 4, the CMFE value range of $I M F_{1}$ in State 2 is 0.0049 0.0180 , and the CMFE value range of $I M F_{1}$ in State 3 is $0.0126-0.0305$, with an intersection of $0.0126-0.0180$. If only the CMFE value of $I M F_{1}$ is used for classification, the disturbance categories within this intersection range cannot be correctly classified, hence three IMF components are used for classification. The CMFE value of $I M F_{2}$ in State 2 ranges from 0.0021 to 0.0043 , while that of $I M F_{2}$ in State 3 ranges from 0.0311 to 0.2566 . Therefore, even if $I M F_{1}$ in States 2 and 3 in the intersection cannot be classified successfully, it can be classified by $I M F_{2}$. By observing and comparing $I M F_{1}, I M F_{2}$, and $I M F_{3}$ in the four states in the table, it can be concluded that the four states can be distinguished by these value classes. For comparison, the parameters of VMD and EEMD are calculated, as shown in Tables 5 and 6, respectively. 
Table 5. CMFE evaluation parameters of VMD in four states.

\begin{tabular}{lcccc}
\hline \multirow{2}{*}{ State } & IMF & \multicolumn{3}{c}{ CMFE of VMD } \\
\cline { 2 - 4 } & & Value Range & Mean Value & Variance \\
\hline \multirow{3}{*}{ State 1 } & $I M F_{1}$ & $0.9901-2.6782$ & 1.7052 & 0.2026 \\
\cline { 2 - 5 } & $I M F_{2}$ & $0.5742-2.6246$ & 0.7339 & 0.1657 \\
\cline { 2 - 5 } State 2 & $I M F_{3}$ & $0.9360-15.6647$ & 4.4749 & 14.7161 \\
\cline { 2 - 5 } & $I M F_{1}$ & $0.0028-0.0491$ & 0.0121 & $7.3699 \times 10^{-5}$ \\
\hline \multirow{3}{*}{ State 3 } & $I M F_{2}$ & $0.0056-1.8155$ & 0.8655 & 0.6079 \\
\cline { 2 - 5 } & $I M F_{3}$ & $0.0179-3.0592$ & 1.7796 & 0.3520 \\
\hline \multirow{nyyyy}{*}{ State 4 } & $I M F_{1}$ & $0.0353-0.1184$ & 0.0535 & $5.1415 \times 10^{-4}$ \\
\cline { 2 - 5 } & $I M F_{2}$ & $1.6848-3.2517$ & 2.4404 & 0.2222 \\
\hline & $I M F_{3}$ & $0.0306-3.9250$ & 1.3662 & 1.3549 \\
\cline { 2 - 5 } & $I M F_{1}$ & $0.2456-0.7048$ & 0.4128 & 0.0074 \\
\hline & $I M F_{2}$ & $0.0048-2.6649$ & 1.3939 & 0.5016 \\
\hline & $I M F_{3}$ & $0.0179-3.0592$ & 1.7796 & 0.3520 \\
\hline
\end{tabular}

Table 6. CMFE evaluation parameters of EEMD in four states.

\begin{tabular}{ccccc}
\hline \multirow{2}{*}{ State } & IMF & \multicolumn{3}{c}{ CMFE of EEMD } \\
\cline { 3 - 4 } & & Value Range & Mean Value & Variance \\
\hline \multirow{3}{*}{ State 1} & $I M F_{1}$ & $1.8885-7.7224$ & 2.4129 & 1.0112 \\
& $I M F_{2}$ & $1.8442-4.4897$ & 2.4984 & 0.3383 \\
& $I M F_{3}$ & $0.9122-19.3505$ & 4.2639 & 16.1200 \\
\hline \multirow{3}{*}{ State 2 } & $I M F_{1}$ & $0.0014-0.0802$ & 0.0252 & $3.5337 \times 10^{-4}$ \\
& $I M F_{2}$ & $0.0016-2.5909$ & 0.9228 & 0.7383 \\
State 3 & $I M F_{3}$ & $0.0133-4.2464$ & 1.7678 & 0.4866 \\
& $I M F_{1}$ & $0.0408-0.1839$ & 0.1088 & 0.0012 \\
State 4 & $I M F_{2}$ & $0.0345-4.1319$ & 2.3524 & 0.5414 \\
& $I M F_{3}$ & $0.0411-4.4743$ & 1.4665 & 1.7213 \\
\hline & $I M F_{1}$ & $0.0029-0.7439$ & 0.4128 & 0.0168 \\
& $I M F_{2}$ & $0.0024-3.6405$ & 1.4189 & 0.7393 \\
& $I M F_{3}$ & $5.0444-9.4536$ & 6.8623 & 0.5020 \\
\hline
\end{tabular}

By observing and comparing the parameters of the three tables, it can be seen that the variance of CMFE calculated by MBMD is the smallest, followed by VMD, and the variance of EEMD is the largest. For the characteristic value of a state, it must be that the smaller the fluctuation, the smaller the variance is, which is better; in addition, the four figures in the previous section, Figures 24-27, also showed the superiority of MBMD in extracting characteristic values. Therefore, the CMFE values of the three IMF components obtained by MBMD were used as samples, and the BP neural network algorithm was used to identify their disturbance categories.

The BP neural network is a feedforward network; it is multi-layered, and it is trained through error back propagation. In order to minimize the error sum of squares of the network, it continuously adjusts the weight and threshold of the network through backpropagation and uses the fastest descent method. We took 30 sets of samples in each state as training samples, and 10 sets as test samples. The BP neural network algorithm was used to classify the test samples. The BP classification diagrams of the three signal decomposition algorithms are shown in Figures 28-30. The classification accuracy rate of each of the four state classifications and the parameters of the total classification accuracy rate are shown in Table 7. 


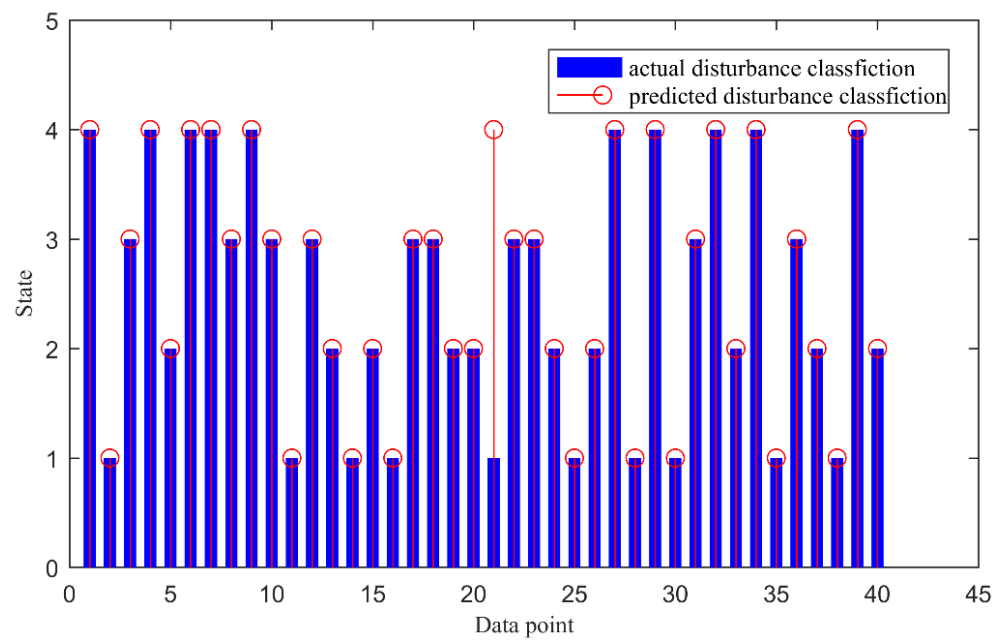

Figure 28. The BP classification diagram of the value of the components decomposed by MBMD calculated by CMFE.

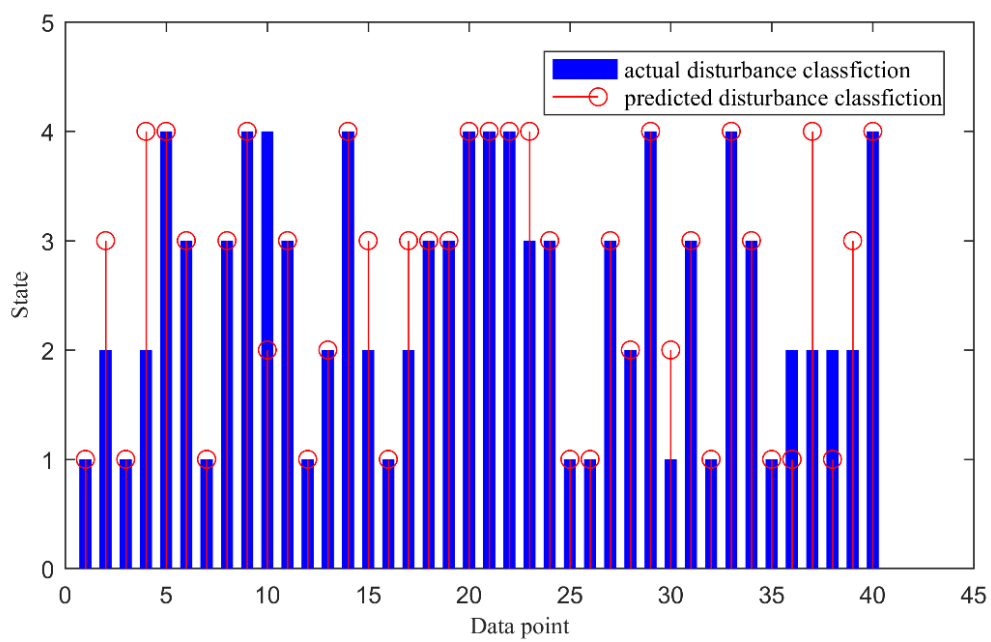

Figure 29. The BP classification diagram of the value of the components decomposed by VMD calculated by CMFE.

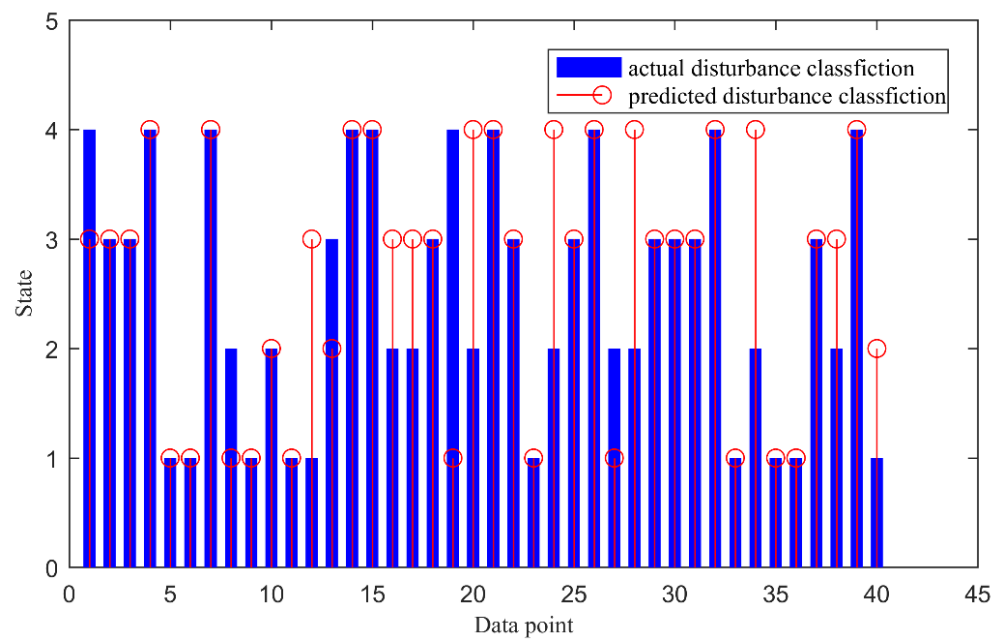

Figure 30. The BP classification diagram of the value of the components decomposed by EEMD calculated by CMFE. 
Table 7. BP classification parameters of the three algorithms.

\begin{tabular}{|c|c|c|c|c|}
\hline \multirow{2}{*}{ State } & \multirow{2}{*}{ Parameter } & \multicolumn{3}{|c|}{ Algorithm } \\
\hline & & MBMD & VMD & EEMD \\
\hline \multirow{2}{*}{ State 1} & Error point & 1 & 1 & 2 \\
\hline & Accuracy & $90 \%$ & $90 \%$ & $80 \%$ \\
\hline \multirow{2}{*}{ State 2} & Error point & 0 & 8 & 9 \\
\hline & Accuracy & $100 \%$ & $20 \%$ & $10 \%$ \\
\hline \multirow{2}{*}{ State 3} & Error point & 0 & 1 & 1 \\
\hline & Accuracy & $100 \%$ & $90 \%$ & $90 \%$ \\
\hline \multirow{2}{*}{ State 4} & Error point & 0 & 1 & 2 \\
\hline & Accuracy & $100 \%$ & $90 \%$ & $80 \%$ \\
\hline \multirow{2}{*}{ Total } & Error point & 1 & 11 & 14 \\
\hline & Accuracy & $97.50 \%$ & $72.50 \%$ & $65.00 \%$ \\
\hline
\end{tabular}

It can be seen in Figure 28 that the predicted classification results of the 40 test samples are highly consistent with the actual categories, while Figures 29 and 30 still illustrate a large number of classification errors. Table 7 lists the specific classification parameters. It can be seen that the MBMD algorithm only misclassified one case in the classification of State 1 , and the classification of other states was 100\% successful, with a total accuracy of $97.50 \%$; on the other hand, the VMD and EEMD algorithms had an unsatisfactory classification effect of only about $70 \%$. It can be seen that the classification effect of these two algorithms for State 1, State 3, and State 4 is still good, both of which are above $80 \%$. However, when State 2 is classified, a large number of errors occur, with only $20 \%$ and $10 \%$ accuracy, respectively. The main reason is that State 2 is a harmonic plus square wave signal, and the two algorithms are not ideal in extracting the features of the other wave signal due to the defects caused by the Gibbs phenomenon or extreme point interpolation. The experimental results show that the BP neural network combined with the MBMD algorithm can effectively identify the disturbance types in photovoltaic DC signals.

\section{Conclusions}

The photovoltaic DC current signal and its disturbance were taken as the research objects, and a series of algorithm experiments on feature extraction and classification was conducted. A new method of identifying the disturbance state of the photovoltaic DC current is consequently proposed.

(1) The categories and causes of photovoltaic DC current signal and its disturbance are proposed to be studied, and the corresponding mathematical model is then constructed. This mathematical model can be used for the simulation of photovoltaic DC signals and other papers about the photovoltaic DC current.

(2) The MBMD method is proposed to solve the problem of broadband signal feature extraction due to the Gibbs phenomenon in the photovoltaic DC signal disturbance analysis. In past time-frequency methods such as EEMD, VMD, and other defects caused by extreme point interpolation or the Gibbs phenomenon, it is proposed that the MBMD method can be avoided by searching in the dictionary. The experimental and simulation results verify that the method can effectively extract the characteristics of broadband components in photovoltaic DC signals. This algorithm can denoise, especially for DC current signals containing square wave signals, and the denoising effect is better than other algorithms.

(3) Combined with the MBMD algorithm, CMFE, and the BP neural network algorithm, a set of recognition methods for photovoltaic DC signal disturbance are proposed. The results show that the recognition accuracy of this method is high, and it can effectively 
identify the disturbance in the photovoltaic DC signal. It can be used as a special method to detect and identify disturbance types in photovoltaic DC signals.

(4) When analyzing complex signals composed of wideband components, the application of MBMD can be extended to signal processing in other fields. However, because the optimization process is more complicated, MBMD takes more time than EEMD and VMD. Therefore, in the future, we will study some fast optimization methods for MBMD and some other improved algorithms.

Author Contributions: Algorithm proposal and innovation, Z.W. and Y.P.; simulation experiment and data analysis, Z.W. and H.G.; experimental supervisors, Y.L. (Yanfei Liu) and Y.G.; writingoriginal draft preparation, Z.W.; equipment debugging and data management, Y.L. (Yi Liu), S.L., and C.F.; writing - review and editing, Z.W., Y.P. and Y.G. All authors have read and agreed to the published version of the manuscript.

Funding: This work was funded by the National Natural Science Foundation of China under Grant No. 51805161, the Research Project of Hunan Provincial Department of Education under Grant No. 19C0769, the Hunan Provincial Natural Science Foundation of China under Grant Nos. 2021JJ30260 and 2018JJ3182, the Scientific Research Fund of Hunan Provincial Education Department under Grant No. 19B191, and the Hunan Innovative Province Construction Special Foundation under Grant No. 2019RS2062.

Institutional Review Board Statement: Not applicable.

Informed Consent Statement: Not applicable.

Data Availability Statement: Data sharing not applicable.

Conflicts of Interest: The authors declare no conflict of interest.

\section{References}

1. Dash, P.K.; Satapathy, P.; Nayak, P.; Sahani, M. Islanding and power quality disturbance monitoring in microgrid using adaptive cross variational mode decomposition and reduced kernel ridge regression. Int. Trans. Electr. Energy Syst. 2020, 30, e12364. [CrossRef]

2. Leite, D.; Decker, L.; Santana, M.; Souza, P. EGFC: Evolving gaussian fuzzy classifier from never-ending semi-supervised data streams-With application to power quality disturbance detection and classification. In Proceedings of the 2020 IEEE International Conference on Fuzzy Systems (FUZZ-IEEE), Glasgow, UK, 19-24 July 2020; pp. 1-9. [CrossRef]

3. Mishra, M. Power quality disturbance detection and classification using signal processing and soft computing techniques: A comprehensive review. Int. Trans. Electr. Energy Syst. 2019, 29, e12008. [CrossRef]

4. López, C.S.P.; Lucchi, E.; Leonardi, E.; Durante, A.; Schmidt, A.; Curtis, R. Risk-benefit assessment scheme for renewable solar solutions in traditional and historic buildings. Sustainability 2021, 13, 5246. [CrossRef]

5. Chen, Z.; Li, M.; Ji, T.; Wu, Q. Real-time recognition of power quality disturbance-based deep belief network using embedded parallel computing platform. IEEJ Trans. Electr. Electron. Eng. 2020, 15, 519-526. [CrossRef]

6. Qiu, W.; Tang, Q.; Liu, J.; Yao, W. An automatic identification framework for complex power quality disturbances based on multi-fusion convolutional neural network. IEEE Trans. Ind. Inf. 2020, 16, 3233-3241. [CrossRef]

7. Satija, U.; Trivedi, N.; Biswal, G.; Ramkumar, B. Specific emitter identification based on variational mode decomposition and spectral features in single hop and relaying scenarios. IEEE Trans. Inf. Forensics Secur. 2019, 14, 581-591. [CrossRef]

8. Lee, S.; Pang, H. Feature extraction based on the non-negative matrix factorization of convolutional neural networks for monitoring domestic activity with acoustic signals. IEEE Access 2020, 8, 122384-122395. [CrossRef]

9. Huang, N.E.; Shen, Z.; Long, S.R.; Wu, M.C.; Shih, H.H.; Zheng, Q.; Yen, N.; Tung, C.C.; Liu, H.H. The empirical mode decomposition and the Hilbert spectrum for nonlinear and non-stationary time series analysis. Proc. R. Soc. A Math. Phys. Eng. Sci. 1998, 454, 903-995. [CrossRef]

10. Yen, G.Y.; Lin, K.C. Wavelet packet feature extraction for vibration monitoring. IEEE Trans. Ind. Electron. 2000, 47, 650-667. [CrossRef]

11. Dragomiretskiy, K.; Zosso, D. Variational mode decomposition. IEEE Trans. Signal Process. 2014, 62, 531-544. [CrossRef]

12. Yang, K.; Wang, G.; Dong, Y.; Zhang, Q.; Shang, L. Early chatter identification based on an optimized variational mode decomposition. Mech. Syst. Signal Process. 2019, 115, 238-254. [CrossRef]

13. Ali, M.; Khan, A.; Rehman, N.U. Hybrid multiscale wind speed forecasting based on variational mode decomposition. Int. Trans. Electr. Energy Syst. 2018, 28, e2466. [CrossRef] 
14. Wei, J.; Xie, T.; Wang, T. A VMD denoising-based imbalance fault detection method for marine current turbine. In Proceedings of the IECON 2020-46th Annual Conference of the IEEE Industrial Electronics Society, Marina Bay Sands Expo and Convention Centre, Singapore, 18-21 October 2020; pp. 1-6. [CrossRef]

15. Sweeney, K.T.; McLoone, S.F.; Ward, T.E. The use of ensemble empirical mode decomposition with canonical correlation analysis as a novel artifact removal technique. IEEE Trans. Biomed. Eng. 2013, 60, 97-105. [CrossRef]

16. Wu, Z.; Huang, N.E. Ensemble empirical mode decomposition: A noise-assisted data analysis method. Adv. Adapt. Data Anal. 2009, 1, 1-59. [CrossRef]

17. Yi, C.; Wang, D.; Fan, W.; Tsui, K.; Lin, J. EEMD-based steady-state indexes and their applications to condition monitoring and fault diagnosis of railway axle bearings. Sensors 2018, 18, 704. [CrossRef] [PubMed]

18. Hao, Y.; Du, Z.; Xing, Z. Leakage signal analysis of urban gas pipeline based on improved variational mode decomposition. Int. J. Pattern Recognit. Artif. Intell. 2020, 34, 1-16. [CrossRef]

19. Ridder, F.D.; Pintelon, R.; Schoukens, J.; Verheyden, A. Reduction of the gibbs phenomenon applied on nonharmonic time base distortions. IEEE Trans. Instrum. Meas. 2005, 54, 1118-1125. [CrossRef]

20. He, K.; Zhang, Z.; Xiao, S.; Li, X. Feature extraction of ac square wave saw arc characteristics using improved hilbert-huang transformation and energy entropy. Measurement 2013, 46, 1385-1392. [CrossRef]

21. Peng, Y.; Li, Z.; He, K.; Liu, Y.; Lu, Q.; Li, Q.; Liu, L.; Luo, R. Broadband mode decomposition and its application to the quality evaluation of welding inverter power source signals. IEEE Trans. Ind. Electron. 2020, 67, 9734-9746. [CrossRef]

22. Peng, Y.; Li, Z.; He, K.; Liu, Y.; Lu, Q.; Li, Q.; Liu, L.; Luo, R. Quality monitoring of aluminum alloy DPMIG welding based on broadband mode decomposition and MMC-FCH. Measurement 2020, 158, 107683-107694. [CrossRef]

23. Zheng, J.; Jiang, Z.; Pan, H. Sigmoid-based refined composite multiscale fuzzy entropy and t-SNE based fault diagnosis approach for rolling bearing. Measurement 2018, 129, 332-342. [CrossRef]

24. Zheng, J.; Pan, H.; Cheng, J. Rolling bearing fault detection and diagnosis based on composite multiscale fuzzy entropy and ensemble support vector machines. Mech. Syst. Signal Process. 2017, 85, 746-759. [CrossRef]

25. Wang, Z.; Peng, Y.; Liu, Y.; He, K.; Wu, Z.; Li, B. The calculation method of pv direct current energy based on modulated broadband mode decomposition and compound simpson integral algorithm. IEEE Access 2021, 9, 51403-51415. [CrossRef] 